\title{
FIELD-THEORETIC TECHNIQUES IN THE STUDY OF CRITICAL PHENOMENA
}

\author{
C. Bagnuls ${ }^{\dagger}$, C. Bervillier ${ }^{\dagger \dagger}$ \\ †Service de Physique de l'Etat Condensée, ${ }^{\dagger \dagger}$ Service de Physique Théorique \\ C. E. A.-Saclay, F91191 Gif-sur-Yvette Cedex, France
}

(Received August 29, 1996)

\begin{abstract}
We shortly illustrate how the field-theoretic approach to critical phenomena takes place in the more complete Wilson theory of renormalization and qualitatively discuss its domain of validity. By the way, we suggest that the differential renormalization functions (like the $\beta$-function) of the perturbative scalar theory in four dimensions $\left(\varphi_{4}^{4}\right)$ should be Borel summable provided they are calculated within a minimal subtraction scheme.
\end{abstract}

Key words: field-theoretic approach, critical phenomena, renormalization group.

PACS number(s): 11.10.Hi, 05.70.Jk

\section{INTRODUCTION}

Field-theoretic techniques have appeared efficient in the obtention of universal properties attached to critical phenomena (for a review see [1]) and they are, nowadays, currently used as such. However these techniques have been questioned by the past (see, e.g., [2-5]) and, recent discussions, relative to some particular aspects of the approach to criticality, such as the sign of the corrections to the Ising scaling [6-9] or the form of the classicalto-Ising crossover [10], have again put forward questions relative to their domain of validity in the study of critical phenomena.

By qualitatively describing the relations between the analytical [11] field theoretic [12-14] and the Wilson [15] formulations of the renormalization group ( $\mathrm{RG}$ ) theory, our aim in this article is to illustrate the origin of the limitations of the former approach. To this end we have considered the local potential approximation [16-18] of the renormalization of the scalar theory [19] that, under the form of a non-linear partial differential equation, involves the essential properties of Wilson's exact equations $[15,20]$.

We stress on the fact that this article is only for illustrative purpose, especially, we do not try to be rigorous (the reader interested in exactness and/or in explicit mathematical formulas may look, for instance, at [21-23,8,9]; other recent studies of the local potential approximation of the RG equations are quoted in [24]). The ingredients of this article are taken from a more detailed version [25], some of them have already been the object of short publications [26-28].

The organization of the article is as follows.

In the following of this part we briefly remind the reader of the main steps of the analytical version of renormalization with a view to introduce our notations and some useful definitions. We then briefly present the questions raised by some authors relatively to the limit of validity of the field-theoretic approach to critical phenomena [14]. In part II, we introduce and formally discuss the notion of continuous scale dependence in field theory which is the main physical consequence of the renormalization theory in, say, particle physics [29]. We illustrate how it is used in the field-theoretic approach to critical phenomena. The object of this part is to bring out some general features of the analytical framework with a view to recover them in the non-analytic approach of Wilson considered in part III. There, we study in some detail the concrete example of the local potential approximation of the exact RG equations. We discuss the continuum limit of interest in field theory with a view to a comparison with the analytical treatment. Especial emphasis is made on the massless (or critical) theory in three dimensions and several notions such as the renormalized coupling "constant", the " $\beta$-function" $\cdots$ are presented. We concretely show that a " $\beta$-function" is associated to the slowest renormalization flow (see [15], p. 132 and [22]) in the critical (massless) hypersurface of the Wilson space of Hamiltonian-parameters. We also concretely show the existence of the slowest renormalization flow approaching the infra-red stable fixed point from the "wrong" side and responsible for "negative" corrections to the Ising scaling [28]. Finally, in part IV, we briefly discuss on the origin of the singularities encountered in perturbation field theory. We especially argue that the perturbative series of the $\beta$-function calculated within a minimal renormalization scheme in four dimensions should be Borel summable.

\section{A. Brief reminder, notations and definitions}

Let us consider the scalar "Hamiltonian" involving the single $\phi_{d}^{4}$ interaction and a momentum cut off $\Lambda_{0}$.

$$
\begin{aligned}
\mathcal{H}\{\phi\} & =\int d^{d} x\left[\frac{1}{2}\left\{(\nabla \phi(x))^{2}+m_{0}^{2} \phi^{2}(x)\right\}\right. \\
& \left.+\frac{g_{0} \Lambda_{0}^{4-d}}{4 !} \phi^{4}(x)\right]
\end{aligned}
$$


The perturbative renormalization process [30] introduces a redefinition of the field $\left(\phi \rightarrow \phi_{\mathrm{R}}\right)$, of the coupling constant $\left(g_{0} \rightarrow u\right)$ and of the mass $\left(m_{0} \rightarrow m\right)$. Subtraction conditions are necessary to fix the finite values of the primary divergent parts of the theory. The arbitrariness in the choice of the finite parts is exploited to give rise to the RG equation [12-14] (see Eq. (1.2)).

It is worth to distinguish two main families of subtraction conditions:

family 1 "subtraction point" schemes that define the finite parts by explicit conditions on some renormalized vertex-functions considered at some value of their external momenta (or wave vectors).

family 2 "minimal subtraction" schemes such as obtained by exclusively subtracting the poles occurring at $d=4$ in the dimensionally regularized perturbation expansion [31]. They amount to selecting a peculiar (minimal or the "slowest", see section III D 1) scale dependence for the renormalized coupling "constant".

In the first family, two types of renormalization schemes must be distinguished:

type 1 the massive schemes, in which the limit of a vanishing renormalized mass $(m \rightarrow 0)$ - i.e., the critical theory - is not defined. In such schemes the mass parameter $m$ is similar to the inverse of the correlation length $\xi$ of statistical systems and provides a "natural" scale of reference for the momenta (or the wave vectors).

type 2 the Weinberg [32] schemes, in which the massless theory is defined. A necessary "arbitrary" scale of reference $(\mu)$ is introduced. The renormalized "mass" parameter $(t)$, when it is different from zero, is similar to the reduced temperature scale $\left(T-T_{\mathrm{c}}\right) / T_{\mathrm{c}}$ of statistical systems undergoing a second order phase transition.

Family 2 involves only schemes of type 2 . For the sake of shortness, from now on, we shall exclusively consider a renormalization scheme of type 2 .

The renormalized vertex (or correlation) functions $\Gamma_{\mathrm{R}}^{(N)}\left(\left\{p_{i}\right\} ; t, u, \mu\right)$ satisfy the following RG equation [14]:

$$
\begin{aligned}
& {\left[\mu \frac{\partial}{\partial \mu}+\beta(u) \frac{\partial}{\partial u}+\left(2-\frac{1}{\nu(u)}\right) t \frac{\partial}{\partial t}-\frac{N}{2} \eta(u)\right]} \\
& \times \Gamma_{\mathrm{R}}^{(N)}\left(\left\{p_{i}\right\} ; t, u, \mu\right)=0
\end{aligned}
$$

in which the differential renormalization functions $\beta(u)$, $\nu(u)$ and $\eta(u)$ are calculable as series in powers of $u$.

The $\beta$-function is of particular interest. It controls the differential evolution of $u$ as the arbitrary scale $\mu$ is varied:

$$
\mu \frac{d u}{d \mu}=\beta(u)
$$

$$
\beta(u)=-(4-d) u+u^{2}+O\left(u^{3}\right)
$$

\section{An implicit hypothesis}

The perturbative expansion of field theory diverges [33] but the proof [34] of Borel summability of the perturbative series of the massive $\phi_{3,2}^{4}$ field theories (in dimension three or two) suggests that the other schemes of renormalization produce also Borel summable series for $d<4[1,35]$. In addition to Borel summability, it is however implicitly assumed that the differential renormalization functions are non-singular at the nontrivial (infra-red stable) fixed point $u_{\mathrm{ir}}^{*}$, i.e.,

$$
\begin{aligned}
& \beta(u)=\omega\left(u-u_{\mathrm{ir}}^{*}\right)+O\left[\left(u-u_{\mathrm{ir}}^{*}\right)^{2}\right], \\
& \nu(u)=\nu+O\left[u-u_{\mathrm{ir}}^{*}\right], \\
& \eta(u)=\eta+O\left[u-u_{\mathrm{ir}}^{*}\right] .
\end{aligned}
$$

\section{Main "objections" encountered in the literature}

1. Parisi has objected [2] that the infra-red singularities involved in the perturbative expansion of the massless theory (set 2) with $d<4$, and which are by-passed by assuming that $\epsilon=4-d$ is an infinitesimal parameter ( $\epsilon$-expansion) [36], do not allow any confidence in the estimations of critical exponents in three dimensions from the massless theory "without an additional hypothesis on the resummation of these infra-red singularities" [2]. Instead Parisi advocates the use of the massive framework (set 1) directly in three dimensions. From another side, it has been shown [37] that the elimination of the infra-red singularities of the massless theory $(d<4)$ requires the explicit consideration of parameters, such as the "critical bare mass" $m_{0 \mathrm{c}}$ of the dimensionally regularized theory. The main consequence of this elimination is the appearance of non-perturbative terms in the expansion [37], thus revealing the non-perturbative nature of field theory [26].

2. Baker and Kincaid [5] have emphasized [38] that the field-theoretic approach to critical phenomena is based on the two limits $\Lambda_{0} \rightarrow \infty$ (continuum limit) and $g_{0} \rightarrow \infty$ (i.e., $u \rightarrow u_{\mathrm{ir}}^{*}$ ) performed in this order, consequently the framework of field theory could be unsuited to describe the critical behavior of some systems especially those which correspond to an infinite $g_{0}$ while $\Lambda_{0}$ is finite (e.g. the spin- $\frac{1}{2}$ Ising model). A discussion of this "objection" has already been presented elsewhere [27] and we shall not reproduced it in this article. 
3. Liu and Fisher [6] and Nickel [7] have pinpointed that the (negative) sign of the corrections to scaling in respectively the three-dimensional Ising models and the self-avoiding walk models is not reproduced in the field theoretic framework. This is rigorously true since the renormalized coupling of the $\phi_{d}^{4}$-theory (with $d<4$ ) is, by construction, confined to the range $\left[0, u_{\mathrm{ir}}^{*}\right][39]$ (see also $[8,9]$ ) inducing a positive sign for the corrections to scaling $[27,40,28]$. However, the notion of effective field theory (see section III D 2) allows us to make sense to calculations in the range $u>u_{\mathrm{ir}}^{*}$ in the renormalized theory $[27,28]$. The detailed discussion of that point being already presented in [28], we shall, again, not reproduce it in this article (see, however, section III E 1).

4. Nickel $[3,4]$ has asserted that the $\beta$-function of the massive scheme in three dimensions involves confluent-branch-point-singularities at the nontrivial fixed point $u_{\mathrm{ir}}^{*}$ implying at least that the quoted uncertainty in the field theoretic estimates of critical exponents are "unrealistically small" (see part IV).

\section{SCALE-DEPENDENCE IN QUANTUM FIELD THEORY}

"Renormalization is not a technical device to get rid of infinities but rather is an expression of the variation of the structure of physical interactions with changes in the scale of the phenomena being probed." [41]

Theories are classified according to the range of length-scales that they cover: classical mechanics, atomic physics, nuclear physics, particle physics. That kind of scale-dependence is step-wise: the physical parameters involved in a theory are constant in the range of lengthscales that the theory is assumed to cover. The scaledependence we are presently interested in differs from the usual notion in that some (or all) physical parameters of a theory are no longer constant but continuously depend on the specific length-scale referred to in the range of validity of the theory. The technique which allows us to define and to deal with such continuously scale-dependent parameters is called the renormalization group first discovered and developed [42] in studying field theory [12,13].

The analytical version of renormalization theory yields the following property for the renormalized two-point vertex function of the massless $(t=0) \varphi^{4}$-theory in four dimensions:

$$
\tilde{\Gamma}_{\mathrm{R}}^{(2)}\left(\frac{p}{\mu} ; u\right)=Z_{3}(\lambda) \tilde{\Gamma}_{\mathrm{R}}^{(2)}\left(\frac{p}{\lambda \mu} ; u(\lambda)\right)
$$

in which we have used $\mu$ to reduce the dimension of the two-point function $\tilde{\Gamma}_{\mathrm{R}}^{(2)}=\Gamma_{\mathrm{R}}^{(2)} / \mu^{2}$.

Eq. (2.8) expresses that $\Gamma_{\mathrm{R}}^{(2)}$ takes on essentially the same form (up to some factor) at external momentum values $\tilde{p}$ and $\tilde{p} / \lambda$ (measured in unit of $\mu$ ) provided that $u$ and the field $\phi_{\mathrm{R}}(x)$ are changed adequately into $u(\lambda)$ and $\phi_{\mathrm{R}}(x) /\left(Z_{3}(\lambda)\right)^{1 / 2}$ respectively.

In Eq. (2.8) the renormalized coupling $u$ is no longer a constant but a function $u(\lambda)$ for which it is meant that $u$ implicitly depends on the global momentum-scale $\mu$ [43].

In field theory, $u$ may be thought of as a physical coupling strength that measures the interaction between scalar particles (if any) and it is unusual to make it continuously depend on a momentum-scale of reference because usual (i.e. at human scales) physical parameters are fixed. That is why one usually refers to $u$ as a coupling "constant". In fact, the continuous scale dependence of the strengths of interactions conveys a fundamental property of field theory [12]. It implies that it is useless to indicate the strength of an interaction if one does not specify the momentum (or energy or length) scale which it is attached to. For example, the usual definition of the electron charge $e\left(\alpha=e^{2} / \hbar c=1 / 137\right)$ by classical macroscopic experiments (like Millikan) corresponds to very large distances [44].

\section{A. Functional form of the scale dependence}

If the parameter $u$ depends on $\mu$ continuously, the analytical version of RG theory does not provide us with the value of $u$ which is actually associated to the given value of the momentum-scale $\mu$ chosen as reference. The analytical approach to field theory (perturbative in essence) only provides us with the evolution of $u$ under a change of $\mu$. For example Eq. (2.8) involves $u(\lambda)$ (corresponding to the change $\mu \rightarrow \lambda \mu$ ) and not the functional form " $u(\mu)$ ". There is an obvious technical reason for this fact: $u$ is dimensionless while $\mu$ is not and in the massless theory no dimensioned parameter other than $\mu$ is available in perturbation. The main reason, however, is that the functional form of the scale dependence is highly nonperturbative in nature (see parts III and IV).

The theoretical $\mu$-dependence of $u$ is, in the analytical treatment, only written down under the differential form via the $\beta$-function [Eq. (1.3)]. An initial condition (an external information such as a measurement) is required to determine the functional form " $u(\mu)$ " from Eq. (1.3). When a value $u_{1}$ has been associated to a given momentum-scale $\mu_{1}$, RG theory allows the determination of all the other values $u(\lambda)$ associated with the running momentum-scale $\mu=\lambda \mu_{1}$ : integration of $\beta(u)$ with the given initial condition, provides the function $u\left(\frac{\mu}{\mu_{1}}\right)=U\left(\mu / \mu_{1} ; u_{1}\right)$. In absence of any initial condition, theory provides us with a continuous family of functions $U$ indexed by all possible values $u_{1}$ that may be associated to a given value of the momentum-scale of reference $\mu_{1}[44]$.

Determining the value of $u$ which is associated to a given value of $\mu$, we call it determining the functional form of the momentum-scale dependence of the coupling strengths. This determination call for non-perturbative methods (see parts III and IV). 


\section{B. A technique to derive critical behavior}

Let us formally illustrate how the momentum-scale dependence of the interaction strengths in QFT is used to study critical behavior.

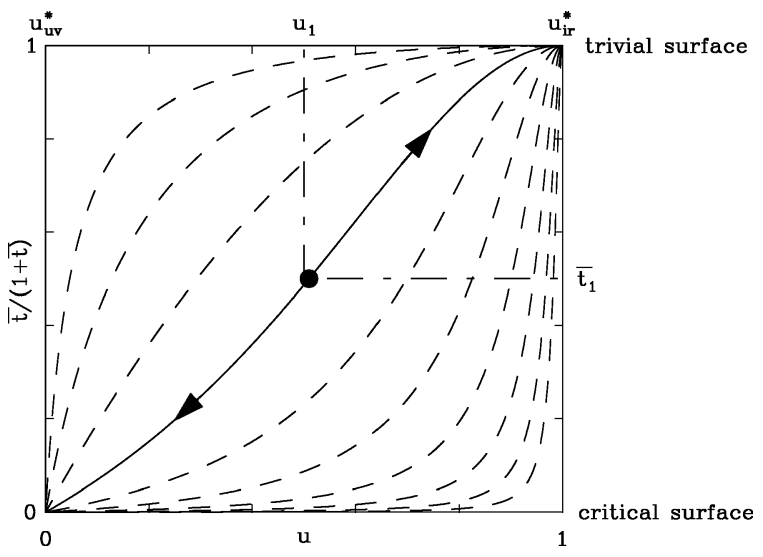

Fig. 1. Qualitative representation of the momentum-scale dependence for the $\phi_{d}^{4}$ field theory with $d<4$. The curves drawn (see also fig. 2) are obtained from assuming $\nu=1 / 2$ and a $\beta$-function of the form $\beta(x)=x(x-1) ; \overline{t(\lambda)}$ stands for $\tilde{t}(\lambda) / \lambda^{2}$. Among the continuous family of curves (dashed curves) a measurement is required in order to choose (full circle) one curve (full curve). An upward arrow indicates the infra-red direction, a downward arrow the ultra-violet direction. See text for more detail.

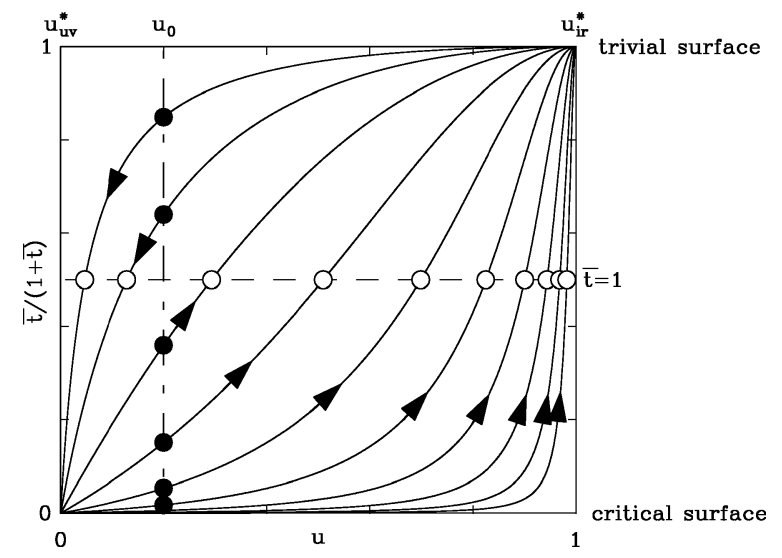

Fig. 2. Use of the $\phi_{d}^{4}$ field theory in the study of critical phenomena. The presentation is similar to fig. 1 . See text for a discussion of the figure.

In order to briefly illustrate the differences which occur in the principles of using renormalization in field theory in one hand and in the study of critical phenomena in the other hand, we have drawn two pictures [figs. 1,2] that qualitatively correspond to the two different frameworks. Let us discuss those two figures.

Field theory: [fig. 1]. Assuming that the "mass"parameter $t$ is actually a measurable quantity, measurements are required in order to fix the particular values $t_{1}$ and $u_{1}$ which are actually associated to the arbitrarily chosen momentum scale $\mu_{1}$. This procedure amounts to selecting an unique (full) curve as indicated on fig. 1.

In absence of an initial condition, there is an infinite family of a priori allowed curves corresponding to the various ways of associating any allowed value of $\bar{t}=t(\lambda) /(\lambda \mu)^{2}$ (in some unit $\mu$ ) to any allowed value of $u$. On fig. 1 we have only drawn a very small number of such allowed curves (dashed curves).

In field theory with $3 \leq d<4$ nothing prevents us from considering the limit $\mu \rightarrow \infty$ (with $\mu=\lambda \mu_{0}$ ) corresponding to an infinite momentum (or energy) scale of reference. It is the ultra-violet regime the direction of which is indicated by a downward arrow on fig. 1 . In this range of momentum scales, masses are negligible and thus any (dashed or full) curve reaches the massless (critical) surface - the lower axis of fig. 1) - at the same point $(\bar{t}=0$ while $u \rightarrow 0)$. This limiting point is the Gaussian fixed point $P_{\mathrm{G}}$ which is ultra-violet stable (for $d<4$ ), we denote it by $u_{\mathrm{uv}}^{*}$. The other fixed point $u_{\mathrm{ir}}^{*}$ [set equal to unity on fig. 1] is only reached in the infra-red limit [small momenta or large distances or low energies, the direction of which is indicated by an upward arrow on fig. 1] provided the mass term is kept fixed to zero at any momentum-scale (massless or critical theory) otherwise the curves go toward the axis $\bar{t}=\infty$. We call this latter axis (upper axis of fig. 1) the "trivial surface" because the physics described in the vicinity of this "surface" corresponds to the classical physics in which the length scales considered are much larger than the relevant length scales (much larger than the Compton wavelength - or than the correlation length as in hydrodynamics). On this "surface" only classical power laws are expected whatever the value of $u$. In particle physics this region corresponds to energies much lower than masses and classical physics is sufficient to describe it. It must be mentioned here that the "trivial surface" may also be considered as a Gaussian fixed point [45] (it is named the infinite mass or the infinite temperature Gaussian fixed point) although only one parameter is actually fixed to a given value (the mass which is infinite).

Statistical physics: [fig. 2]. The use of renormalization theory in studying critical phenomena is, in nature, very different from the previous description. A given physical system corresponds there to a given value of $u$, say $u_{0}$, associated to a given microscopic length scale $1 / \mu_{0}$ (small compared to the correlation length of the system). The vertical axis at $u=u_{0}$ on fig. 2 intersects the RG flows (full curves) at points (full circles) corresponding to different values of $\bar{t}$. These points are representative of the same statistical system ( $u_{0}$ is fixed) observed at different temperatures (measured by reference to the critical temperature of the system considered). For a definite and sufficiently small temperature, say $\bar{t}_{0}<1$, a unique full curve is selected. By following that curve up to $\bar{t}=1$ (up to the open circle on the selected curve as indicated by black arrows), one performs an average of the physics occurring at length scale smaller than $1 /\left(\lambda \mu_{0}\right)$ (which is larger and larger as $\lambda \rightarrow 0$ ). One thus obtains a description of a new physical system (new values of $u$ and of the field $\left.\varphi_{\mathrm{R}}\right)$. This corresponds to the Wilson step of thin- 
ning out the degrees of freedom of the original statistical system. The effective system so obtained is no longer the original one, but the physics occurring at distances larger than or equal to $1 /\left(\lambda \mu_{0}\right)$ remains unchanged compared to the initial system.

By considering smaller and smaller values of $\bar{t}_{0}$, one selects other full curves on fig. 2 on which the full circles approach the critical surface while the open circles approach the axis $u=u_{\mathrm{ir}}^{*}$. Hence the limit $\bar{t}_{0} \rightarrow 0$ at fixed $u_{0}$ is equivalent to the limit $u \rightarrow u_{\mathrm{ir}}^{*}$ at fixed $\bar{t}$ provided one is only interested in sufficiently large distances. Notice that the approach to $u_{\text {ir }}^{*}$ occurs whatever the value of $u_{0}$ and, more importantly, for small enough values of $\bar{t}_{0}$, the full curves accumulate in the vicinity of the axis $u=u_{\mathrm{ir}}^{*}$ along essentially a unique curve departing from the critical surface $\bar{t}=0$. This is a qualitative illustration of universality in critical phenomena.

\section{A NON-PERTURBATIVE EXAMPLE OF RENORMALIZATION GROUP EQUATIONS}

"Even if one succeeds in formulating the renormalization group approach for a particular problem, one is likely to have to carry out a complicated computer calculation, which makes most theoretical physicists cringe." [46]

The main difficulty in understanding the connection between the analytical and Wilson approaches to renormalization relies upon the number of parameters involved: a very small number in one hand (essentially the coupling constant - renormalized or not) and a very large (infinite) number in the other hand.

In order to concretely compare Wilson's theory to the analytical field theory briefly referred to in part II, we consider the differential RG equations of Wegner and Houghton [20] in the local-potential-approximation that allows us to reduce the original infinite set of coupled differential equations of $[15,20]$ to a single partial differential equation (see Eq. (3.11)) for a simple function of the scalar field $\phi[16,17]$.

\section{A. Brief presentation}

Let us consider a general Hamiltonian $\mathcal{H}$ depending on a scalar field $\phi$. It is written as a sum of a Gaussian part and a potential which, in general, is non-local (i.e. involving derivatives of $\phi(x)$ that we symbolically denote by $\partial \phi)$ :

$$
\mathcal{H}=\int d^{d} x\left[\frac{1}{2}(\partial \phi)^{2}+V(\phi, \partial \phi)\right]
$$

The local potential approximation amounts to considering the restriction of the Wilson space $\mathcal{S}$ of Hamiltonian-parameters to a smaller space $\mathcal{S}^{\prime}$ such that $V(\phi, \partial \phi)$ in Eq. (3.9) reduces to its local part $V(\phi)$. Assuming the $O(1)$-symmetry $\phi \rightarrow-\phi$, we thus have for small $\phi$ :

$$
V(\phi)=\sum_{k=1}^{\infty} a_{k} \phi^{2 k}
$$

It is worth to indicate here that the local potential approximation still involves essential properties of the "exact" RG equations $[15,20]$ since it is similar [18] to a continuous version of both the approximate recursion relation introduced in [15] and the hierarchical model of [47].

For the sake of notational simplicity, we denote by $y$ the dimensionless field $\tilde{\phi}=\Lambda_{0}^{(2-d) / 2} \phi$ and we shall no longer distinguish between $\mathcal{S}$ and $\mathcal{S}^{\prime}$.

The Wegner-Houghton RG equations in the local potential approximation reduce to a non-linear partial differential equation $[16,17]$ for $f(y, l)=\partial V(y, l) / \partial y$ with $l$ related to the change of momentum-scale $\Lambda_{0} \rightarrow \Lambda_{l}=$ $e^{-l} \Lambda_{0}$ (in the following we also denote the running momentum-scale $\Lambda_{l}$ by $\Lambda$ ). By analogy with an actual flow in usual space, we shall refer to $l$ as the "time" variable.

Defining $f^{\prime}=\partial f / \partial y, f^{\prime \prime}=\partial^{2} f / \partial y^{2}, \dot{f}=\partial f / \partial l$, the partial differential equation then reads [17]:

$$
\dot{f}=\frac{K_{d}}{2} \frac{f^{\prime \prime}}{1+f^{\prime}}+\left(1-\frac{d}{2}\right) y f^{\prime}+\left(1+\frac{d}{2}\right) f
$$

in which $K_{d}$ is the surface of the $d$-dimensional unit sphere divided by $(2 \pi)^{d}$.

Eq. (3.11) for $\dot{f}=0$ is the fixed point equation. Its numerical study yields the following results. Apart from the trivial Gaussian fixed point $P_{\mathrm{G}}$ (corresponding to $f \equiv 0$ ), one observes the appearance of one new non-trivial fixed point below each dimensional threshold $d_{k}=2 k /(k-1)$, $k=2,3, \ldots, \infty$. In particular, there is only one nontrivial fixed point at $d=3$ and none at $d=4[17,18,26]$.

Strategy adopted in the present study. We consider Eq. (3.11) at $d=3$, which is the dimension referred to in the following (except in section III F where $d=4$ ).

Our aim is to visualize, for the sake of a qualitative comparison with the analytical field theory, the renormalization flows in the Wilson space $\mathcal{S}$ of Hamiltonianparameters [the coordinates of which are given by the coefficients $a_{k}$ of Eq. (3.10)].

Given an initial simple function, say:

$$
f(y, 0)=r_{0}(0) y+u_{0}(0) y^{3}+v_{0}(0) y^{5}
$$

corresponding to a point of coordinates $\left(r_{0}(0), u_{0}(0), v_{0}(0), 0,0, \cdots\right)$ in $\mathcal{S}$, and after having numerically determined the associated solution $f(y, l)$ of Eq. (3.11) at a varying "time" $l$, we concretely represent the Wilson trajectories (entirely plunged in $\mathcal{S}$ ) by numerically evaluating the following derivatives:

$$
r_{0}(l)=\left.\frac{\partial f(y, l)}{\partial y}\right|_{y=0},
$$




$$
\begin{aligned}
& u_{0}(l)=\left.6 \frac{\partial^{3} f(y, l)}{\partial y^{3}}\right|_{y=0}, \\
& v_{0}(l)=\left.120 \frac{\partial^{5} f(y, l)}{\partial y^{5}}\right|_{y=0},
\end{aligned}
$$

etc...

We then are able to visualize the actual Wilson trajectories by means of a projection onto the planes $\left\{r_{0}, u_{0}\right\}$ or $\left\{u_{0}, v_{0}\right\}$ (for example) of $\mathcal{S}$.

At $d=3$ we found the non-trivial infra-red stable fixed point $\left(f^{*}(y)\right)$ located in $\mathcal{S}$ at $r_{0}^{*}=-0.461533 \cdots$, $u_{0}^{*}=3.27039 \cdots, v_{0}^{*}=14.4005 \cdots, w_{0}^{*}=32.31289 \cdots$, etc. This is the fixed point to which refers the parameter $u_{\mathrm{ir}}^{*}$ of the analytical version of RG theory. With a view to illustrate this assertion, we focus our attention on the massless - or critical - theory in which the infra-red stable fixed point is reached when decreasing the momentum scale of reference down to zero.

\section{B. Determination of the critical surface: the "shooting method"}

In order to look at the critical renormalization flows, we must adjust the initial point to be on the critical surface $\mathcal{S}_{c}$. To this end we use the "shooting" method.

As initial function at $l=0$, we consider Eq. (3.12) with $v_{0}(0)=0$. To start on $\mathcal{S}_{\mathrm{c}}$ with, say, $u_{0}(0)=3$, we must adjust $r_{0}(0)$ [or any other Hamiltonian parameter different from $\left.u_{0}(0)\right]$ to a non-zero value [48]. For any choice of an initial function at $l=0$ on the critical surface $\mathcal{S}_{\mathrm{c}}$, there is a particular value $r_{0 \mathrm{c}}(0)=r_{0 \mathrm{c}}\left(u_{0}(0), v_{0}(0), \cdots\right)$ of $r_{0}(0)$ for which the nontrivial fixed point is reached when $l \rightarrow \infty$.

The "shooting" method is based on the fact that, for sufficiently large values of $l$, the renormalization trajectories goes away from $f^{*}$ in two different directions according to the sign of the difference $r(0)-r_{0 c}(0)$.

By numerically determining the values of $r_{0 c}(0)$ corresponding to different initial points in $\mathcal{S}_{\mathrm{c}}$, we are able to visualize the Wilson trajectories approaching $f^{*}$ in $\mathcal{S}_{c}$.

\section{Critical-renormalization-flows and massless field theory in three dimensions}

Some [49] trajectories in $\mathcal{S}_{c}$ are represented on fig. 3 as a projection onto the plane $\left\{u_{0}, v_{0}\right\}$ [50]. Let us discuss this figure.

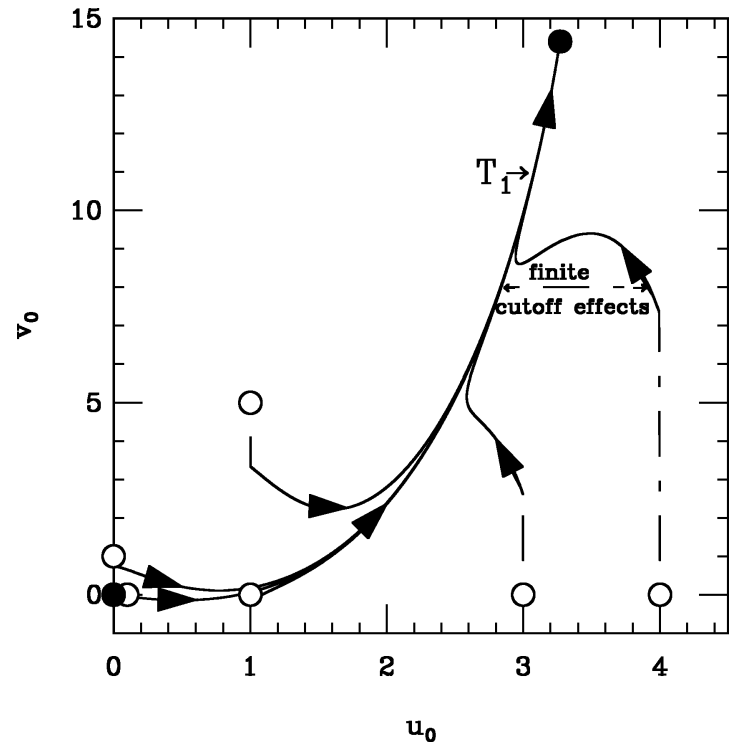

Fig. 3. Projection onto the plane $\left(u_{0}, v_{0}\right)$ of some Wilson's trajectories on the critical surface $\mathcal{S}_{\mathrm{c}}$ (after integration of Eq. (3.11) at $d=3$ ). Full circles indicate the locations of the Gaussian $\left(P_{\mathrm{G}}\right)$ and the non-trivial $\left(f^{*}\right)$ fixed points respectively. Arrows indicate the infra-red direction. Open circles indicate the locations of the initial points. The values of the initial sets of coordinates $\left(r_{0 c}(0), u_{0}(0), v_{0}(0)\right)$ are the following: $(-0.014175577 \cdots, 0.1,0),.(-0.116887403 \cdots$,

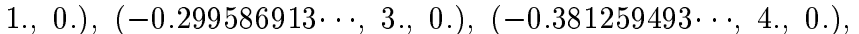
$(-0.180075418 \cdots, 1 ., 5),.(-0.025823782 \cdots, 0 ., 1$.$) the other$ coordinates being set equal to zero. The full curves represent Wilson's trajectories entirely plunged in $\mathcal{S}$. The "vertical pieces" (dot-dashed lines) are numerically undetermined parts of the projected Wilson trajectories. They reflect the lack of accuracy in our determination of $v_{0}(l)$ for small $l$.

Apart from the fact that each Wilson trajectory goes toward the fixed point $f^{*}$ [full circle on the top of fig. 3], one observes that they all approach it asymptotically along the same one-dimensional submanifold in $\mathcal{S}_{c}$. This limiting submanifold, that we denote by $T_{1}$, has its source at the Gaussian fixed point $P_{\mathrm{G}}$. Moreover, the closer the initial points are chosen to $P_{\mathrm{G}}$ [full circle on the bottom of fig. 3], the longer is the "time" that the resulting flows take to go along the unique submanifold. $T_{1}$ is an attractive (infra-red stable) submanifold along which the flow are slowly running (see section III D 1).

According to Wilson's terminology, $T_{1}$ is a renormalized "trajectory" on which is defined the genuine "continuum limit" [51] of field theory. Hence, to make sense, the RG flow of the renormalized massless $\phi_{3}^{4}$ field theory, referred to in part II, must be defined relatively to $T_{1}$.

Because one practically only encounters in the literature the (sketchy) description of the massive case as it is presented in section 12.2 of [15] (i.e., the field theory involving only a mass as renormalized parameter), we find it worthwhile to examine and discuss the procedure of "making the cutoff infinite on $T_{1}$ " (the massless case) in some detail.

By analogy with the analytical treatment that refers to a single coupling "constant", the procedure consists 
in showing that some non-zero "renormalized" parameter (a coupling "constant" for the massless scalar theory) exists when the initial cutoff tends to infinity.

\section{The continuum limit of the massless case $(d=3)$}

The main difference between the well known limit briefly described in [15] and the case we are considering in $\mathcal{S}_{\mathrm{c}}$ is that the final ("renormalized") parameter is not a mass-like parameter (because we are confined to the critical surface) but a coupling-"constant"-like parameter.

Instead of considering surfaces of constant correlation lengths in $\mathcal{S}$, as in the massive case of [15], we consider planes of constant $u_{0}$ (see section III D 3 ).

Let us select a plane of constant $u_{0}$, say $u_{0}=\bar{u}_{0}=3$, orthogonal to the $u_{0}$-axis of $\mathcal{S}$. Let us, then, consider initial points on $\mathcal{S}_{\mathrm{c}}$ such that $0<u_{0}(0)<3$ (the other coordinates being set equal to zero, but $r_{0 \mathrm{c}}(0)$ of course). If $u_{0}(0)=2$, the corresponding Wilson flow intersects the plane $\bar{u}_{0}(=3)$ at $Q_{2,3}$ after some finite "time" $l_{2,3}$. Let us denote by $\mu_{0}$ the effective momentum scale of reference associated to the plane of reference $\bar{u}_{0}=3$ (at present: $\left.\mu_{0}=e^{-l_{2,3}} \Lambda_{0}\right)$ and let us consider it as the new fixed unit of momentum [52]. We then choose another initial point lying closer to $P_{\mathrm{G}}$, say $u_{0}(0)=1$. Compared to $Q_{2,3}$, the new intersection-point $Q_{1,3}$ with the plane of reference is found closer to $T_{1}$ while $l_{1,3}>l_{2,3}$. This means that, $\mu_{0}$ being fixed [53], the initial cutoff $\Lambda_{0}$ "appears to be larger" at $Q_{1,3}$ than it is at $Q_{2,3}$. By considering a sequence of initial points $u_{0}(0)=\alpha$ with $\alpha \rightarrow 0$ (hence approaching the Gaussian fixed point $P_{\mathrm{G}}$ ) the sequence of points $Q_{\alpha, 3}$ on the plane of reference $\bar{u}_{0}=3$ would finally hit $T_{1}$ at $Q_{0,3}$. At this point, $\mu_{0}$ being kept arbitrarily fixed, the initial cutoff $\Lambda_{0}$ "appears to be infinite" since $l_{\alpha, 3} \rightarrow \infty$ when $\alpha \rightarrow 0$ (due to the ultra-violet stability of $P_{\mathrm{G}}$ ). A non-zero value of a (renormalized) " $\phi^{4}$ coupling-constant" (here $\bar{u}_{0}=3$ ) exists and may thus be associated to an arbitrary (finite) momentum scale $\mu_{0}$ in the limit of infinite cutoff $\left(\Lambda_{0} / \mu_{0} \rightarrow \infty\right)$.

Frequently, one ends the discussion at this point being satisfied by the existence of the "infinite-cutoff limit" involving a single non-vanishing renormalized coupling "constant" (here: $\bar{u}_{0} \neq 0$ ). However the analysis is physically incomplete: what about the momentum-scale dependence of QFT discussed in part II ? Where is the effect of the infinite number of degrees of freedom hidden in the limit?

\section{Momentum-scale dependence in the continuum limit}

A quick inspection of the procedure just-abovedescribed shows that one may associate any point of $T_{1}$ (but the ends) to an arbitrary $\mu_{0}$ chosen in the range ] $0, \infty[$. It is usual to say that the massless field theory involves only one "free" parameter (presently, a coupling constant, $\bar{u}_{0}$, the actual value of which lies somewhere in the range $\left.\left[0, u_{0}^{*}=3.27039 \cdots\right]\right)$. But no information on the momentum-scale dependence of $\bar{u}_{0}$ results from these considerations.

The concept of momentum-scale dependence in QFT requires to specify the succession of points of $T_{1}$ associated with the running momentum-scale $\mu=\lambda \mu_{0}$ (with $\lambda$ varying in the range $[0, \infty])$ knowing that some given point ( say $\bar{u}_{0}=3$ ) was primarily associated with the fixed momentum-scale $\mu_{0}$.

After having reached the plane $\bar{u}_{0}=3$, a Wilson flow running closely along $T_{1}$ continues to go toward $f^{*}$. It intersects the plane $\bar{u}_{0}=3.2$, for example, at a momentum scale $\mu_{0}^{\prime}<\mu_{0}$.

Unicity of the differential form: the $\beta$-function. For reasons of continuity, both of $T_{1}$ and of the change of momentum-scale along $T_{1}$, one may easily establish that two points of $T_{1}$, chosen infinitesimally close to each other and identified by their projections ( say $\bar{u}_{0}=3$ and $\left.\bar{u}_{0}=3+d \bar{u}_{0}\right)$ on the $u_{0}$-axis of $\mathcal{S}$, are associated to an infinitesimal change of the momentum-scale of reference $\lambda \mu_{0} \rightarrow(\lambda+d \lambda) \mu_{0}$ such that:

$$
\lambda \frac{d \bar{u}_{0}}{d \lambda}=f_{1}\left(\bar{u}_{0}\right)
$$

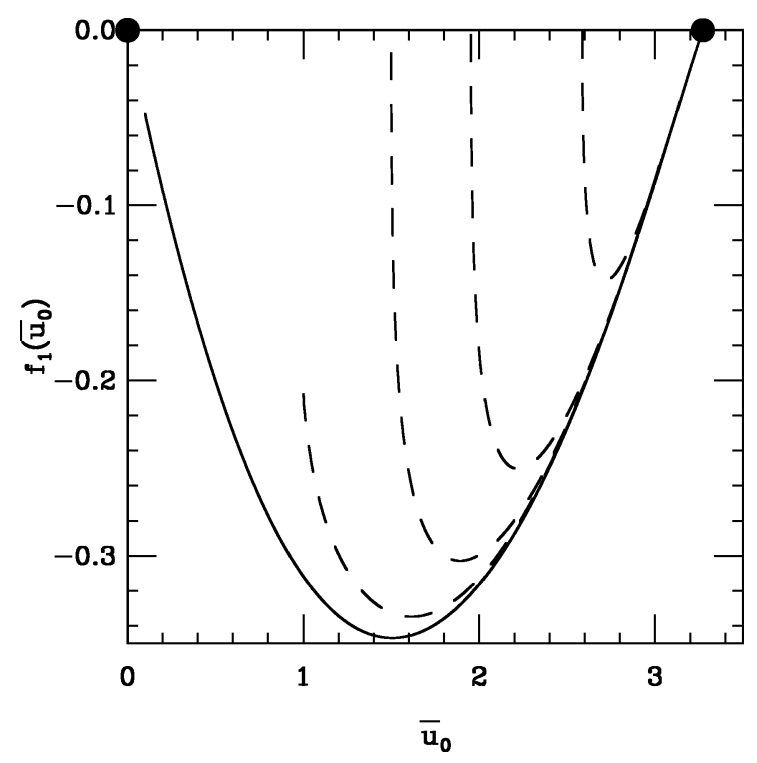

Fig. 4. Graphical representation of the Wilson differential flows (dashed curves), in $\mathcal{S}_{\mathrm{c}}$ at $d=3$, projected on the $u_{0}$-axis - i.e. $f_{1}\left(\bar{u}_{0}\right)=-d \bar{u}_{0}(l) / d l$. In the infra-red direction, any (dashed) curve exponentially approaches a limiting (full) curve which coincides with the differential-momentum-scale-dependence carried by the ultimate Wilson flow emerging from the Gaussian fixed point $P_{\mathrm{G}}$ (lefthand full circle). The function $f_{1}\left(\bar{u}_{0}\right)$ is numerically determined along this flow corresponding to the trajectory $T_{1}$ in $\mathcal{S}_{\mathrm{c}}$. Notice that the convergence is numerically well controlled independently of whether the initial point is chosen close to $P_{\mathrm{G}}$ or not. The righthand full circle indicates the infra-red stable fixed-point-value $u_{0}^{*}=3.27039 \cdots$. 
where the function $f_{1}$ is unique for a given representation of the renormalized coupling (here a projection of the flow onto the $u_{0}$-axis, see section IIID 3). This unicity is illustrated by fig. 4 .

The differential expression of the flow along $T_{1}$ depends on the representation of the renormalized coupling. Many different representations are allowed as discussed in section III D 3. But any representation refers to a unique flow ("on" $T_{1}$ ). Universal features are thus expected, such as in the asymptotic approach to $u_{0}^{*}$ :

$$
f_{1}\left(\bar{u}_{0}\right)=\omega_{1}\left(\bar{u}_{0}-u_{0}^{*}\right)+O\left[\left(\bar{u}_{0}-u_{0}^{*}\right)^{2}\right]+O(0)
$$

in which $\omega_{1}$ is a characteristic feature of the Wilson flows along $T_{1}$ in the vicinity of $f^{*}$ [54]. The term $O(0)$ is present in Eq. (3.17) in order to emphasize that, in principle, the flow of interest runs along the trajectory $T_{1}$ which is entirely plunged in a space of infinite dimension. Thus, one may expect that an infinite number of conditions on the initial Hamiltonian [55,56] should be specified. Actually, the definition of the differential flow does not require this initial condition to be specified provided one is able to select directly the particular flow running along $T_{1}$ (see section IIID 1 and part IV).

Apart the term $O(0)$, the function $f_{1}\left(\bar{u}_{0}\right)$ is like the $\beta$-function of the analytical approach. Only a difference in the choice of parameterizing $T_{1}$ (via $\bar{u}_{0}$ instead of $u$ ) occurs (see section IIID 3 ).

Degeneracy of the functional form: Contrary to the differential form, the determination of the functional form of the scale dependence requires to specify explicitly an initial point on $T_{1}$ (see section II A).
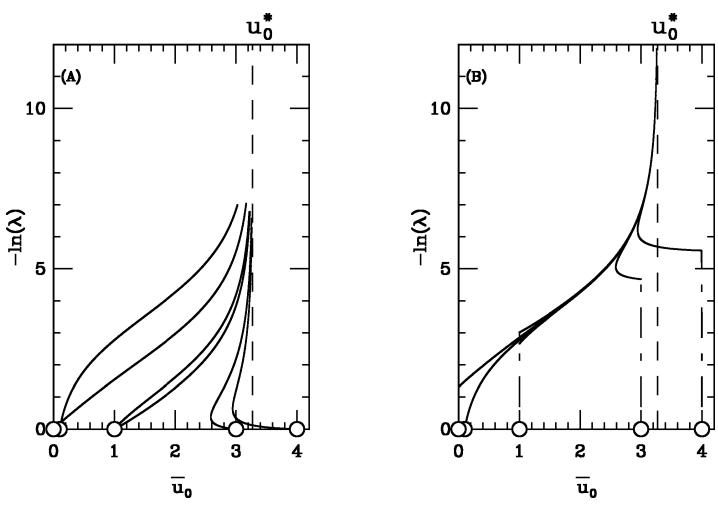

Fig. 5. Functional momentum-scale dependence displayed by $\bar{u}_{0}(l)$ (projection onto the $u_{0}$-axis of $\mathcal{S}$ ) along definite Wilson's flows on $\mathcal{S}_{\mathrm{c}}(\mathrm{A})$. Open circles indicate the initial points chosen in $\mathcal{S}_{\mathrm{c}}$. Each full curve in $(\mathrm{A})$ provides a determination of the functional momentum-scale dependence. In order to emphasize where the effective field theory (see section IIID2) practically occurs in each case, on figure (B) we have artificially translated the "time" scales $l=-\ln (\lambda)$ (vertical dashed lines) such that each actual Wilson's flow of (A) "hits" a given unique value of $\bar{u}_{0}$ at the same "time". The unique functional flow so obtained illustrates the underlying unique differential flow of fig. 4 .
Because one cannot specify the infinite set of coordinates of a particular point lying on $T_{1}$, one arbitrarily chooses an initial point in $\mathcal{S}_{\mathrm{c}}$ in the close vicinity of $P_{\mathrm{G}}$ [57]. This step corresponds to "fine-tuning" the parameters of the initial Hamiltonian (associated to the momentum-scale $\Lambda_{0}$ ). Due to the freedom in adjusting the initial point near $P_{\mathrm{G}}$, an infinite number of definite flows (Wilson's flows) accumulate "on" $T_{1}$. This infinite number indicates the unlimited different possibilities of associating one point of $T_{1}$ (one value of $\bar{u}_{0}$ ) to a fixed $\mu_{0}$ measured in the unit $\Lambda_{0}$ [58].

Any "fine-tuning" provides us with one determination of the functional form of the momentum-scale dependence in the "infinite cutoff limit" which, thus, is degenerated on $T_{1}$. This is illustrated on fig. 5 .

\section{The "large river effect" and the notion of "effective" field theory}

A rather close approach to the unique differential flow numerically determined by reference to the submanifold $T_{1}$ occurs independently of whether the initial point is chosen close to the ultra-violet stable fixed point $P_{\mathrm{G}}$ or not. The convergence towards $T_{1}$ is exponentially rapid and is sufficient to allow a rather correct numerical determination of an unique differential flow for a small enough running momentum-scale of reference.

Indeed $T_{1}$ is like a large river (having its source "at" $P_{\mathrm{G}}$ ) into which watercourses (Wilson's flows) run. (This notion has recently been used for studying the mechanism of a "mimicry" of second-order phase transitions by fluctuation-induced first-order phase transitions [59].)

The 'large river' effect reveals the existence of a deep valley in $\mathcal{S}_{\mathrm{c}}$ where the renormalization flows are slowly varying (see [15], p. 132 and [22]).

It is interesting to note that the exponentially rapid approach to the renormalized trajectory is not indicated in the usual (sketchy) graphical representations of the process of constructing the continuum limit in the Wilson framework (see, for instance, fig. 1 of [8]).

\section{The flows along the "renormalized trajectory" are slowly varying}

Let us consider a definite Wilson flow on $\mathcal{S}_{\mathrm{c}}$ not initialized in the close vicinity of $P_{\mathrm{G}}$ but approaching $f^{*}$ asymptotically along $T_{1}$, for example the flow initialized at $u_{0}(0)=4, v_{0}(0)=0$ and $r_{0 c}(0)=-0.381259493 \cdots$ [see the corresponding trajectory projected in fig. 3]. Using obvious notations we shall refer to this flow by $(4,0)$.

Such kinds of flow may be decomposed into several parts well separated by short transitory ranges of "time". Disregarding small transitory parts, each part of a Wilson trajectory is characterized by a regime of flowvelocities that becomes slower and slower as the part considered is chosen closer and closer to $T_{1}$. On fig. 3 the full curve corresponding to the trajectory $(4,0)$ clearly displays two regimes [60]. 


\section{BAGNULS, C. BERVILLIER}

In order to quantitatively illustrate how the different regimes of flow-velocities evolve along a Wilson trajectory, it is convenient to consider the expected analytic form of the effective momentum-scale dependence of a Wilson flow in $\mathcal{S}_{\mathrm{c}}$ and in the vicinity of $f^{*}$. This gives the following expansions [61]:

$$
\begin{aligned}
& r_{0}(l) \stackrel{l \rightarrow \infty}{\simeq} r_{0}^{*}+b_{r}^{(1,1)} \exp \left(-l \omega_{1}\right)+b_{r}^{(1,2)} \exp \left(-2 l \omega_{1}\right)+\cdots+b_{r}^{(2,1)} \exp \left(-l \omega_{2}\right)+b_{r}^{(2,2)} \exp \left(-2 l \omega_{2}\right)+\cdots \\
& +c_{r}^{(1,2)} \exp \left[-l\left(\omega_{1}+\omega_{2}\right)\right]+\cdots \cdots+\cdots, \\
& u_{0}(l) \stackrel{l \rightarrow \infty}{\simeq} u_{0}^{*}+b_{u}^{(1,1)} \exp \left(-l \omega_{1}\right)+b_{u}^{(1,2)} \exp \left(-2 l \omega_{1}\right)+\cdots+b_{u}^{(2,1)} \exp \left(-l \omega_{2}\right)+b_{u}^{(2,2)} \exp \left(-2 l \omega_{2}\right)+\cdots \\
& +c_{u}^{(1,2)} \exp \left[-l\left(\omega_{1}+\omega_{2}\right)\right]+\cdots \cdots+\cdots, \\
& v_{0}(l) \stackrel{l \rightarrow \infty}{\simeq} \text { etc } \cdots
\end{aligned}
$$

in which $\omega_{n}>\omega_{n-1}>\cdots>\omega_{2}>\omega_{1}$.

The smallest exponent $\omega_{1}$ controls the rate of Wilson's flows in the vicinity of $f^{*}$, it is a characteristic feature of $T_{1}$ in the vicinity of $f^{*}$. In the field-theoretic approach to critical phenomena only one correction-toscaling exponent, usually denoted by $\omega$, is available. In principle $\omega_{1}$ and $\omega$ coincide [62]. Any representation of the momentum-scale dependence "on" $T_{1}$ in the vicinity of $f^{*}$ reduces Eq. (3.19) (projection onto the $r_{0}$-axis) and Eq. (3.20) (projection onto the $u_{0}$-axis) to their respective first parts, the other exponents $\left(\omega_{n}\right.$ with $\left.n>1\right)$ disappear. The correction terms (in the vicinity of $f^{*}$ ) associated to $\omega_{n}$ with $n>1$ are essentially suppressed in Wilson's flows initialized in the close vicinity of $P_{\mathrm{G}}$ or are extremely (exponentially) lessened by decreasing the actual momentum-scale of reference (due to the 'largeriver' effect). They are merely neglected in the analytical treatment. When non-exponentially-small compared to the ideal flow associated to $\omega$, they carry what may be (roughly) called the "finite-cutoff effects".

\section{Notion of effective field theory}

The observation of the "large river effect" leads directly to the notion of "effective field theories" (see, for example, in [63]): a reference to the ultra-violet stable fixed point is not needed to concretely evaluate (within an acceptable accuracy) the behavior of a field theory below some momentum-scale of reference. This notion is more significant when no ultra-violet stable fixed point is available (see sections III E and III F).

\section{The renormalized coupling "constant"}

In sections III C 2 , a projection of $T_{1}$ onto the $u_{0}$-axis has been used to refer to the differential form of the momentum-scale dependence along $T_{1}$ (representation of the momentum-scale dependence "on" $T_{1}$ by means of a single parameter). The associated scale dependent parameter has been denoted by $\bar{u}_{0}$ and called the renormalized parameter. One must not make a confusion between $\bar{u}_{0}$ and the unrenormalized $\phi^{4}$ coupling constant $u_{0}(0)$. An unrenormalized coupling is attached to an initial point lying on some canonical surface of $\mathcal{S}$ (i.e. it refers to an Hamiltonian involving a limited number of parameters). The question of dealing with the infinite number of degrees of freedom has not yet been addressed (a Wilson transformation has not yet been performed). Instead, in the analytical framework, a renormalized parameter is defined by reference (under a differential form) to the actual momentum scale dependence displayed along the attractive submanifold $T_{1}$ (that is plunged into a space of infinite dimension) [64].

The choice we have made of referring to $T_{1}$ by a projection onto the $u_{0}$-axis could appear arbitrary. In particular, it seems that we could have chosen any other axis such as $v_{0}, w_{0}, \cdots$ associated with the Hamiltonianterms $\phi^{6}, \phi^{8}, \cdots[21]$. This is true as long as one is only interested in the momentum-scale dependence along $T_{1}$ away from $P_{\mathrm{G}}$. In this region the slow variation with the momentum-scale of reference of any running Hamiltonian-parameter is a characteristic feature of $T_{1}$. In this respect, the $\phi^{4}$ Hamiltonian-term does not distinguish itself from the other Hamiltonian-terms. We may then choose any Hamiltonian-parameter (or any combination of Hamiltonian-parameters) to describe the differential form of the momentum-scale dependence along $T_{1}$. This is not so in the vicinity of $P_{\mathrm{G}}$ however. There, $T_{1}$ is tangent to the $u_{0}$-axis (see fig. 3 ) and any other 
choice of axis is prohibited otherwise the projection of $T_{1}$ would be empty. The $\phi^{4}$-coupling is called the relevant [65] parameter at the once infra-red unstable fixed point $P_{\mathrm{G}}$. This is why the $\phi^{4}$ Hamiltonian-term plays an essential role in perturbation field theory. Although, farther away from $P_{\mathrm{G}}$ (in a non-perturbative region) this choice appears arbitrary, it is the only choice that can describe the momentum-scale dependence in the whole range of scales from $\infty$ to 0 [66].

From now on, the origin of the freedom in the definition of the renormalized parameter $u$ in perturbation theory (freedom in the choice of the subtraction scheme) may well be understood [17]. Even in the perturbative region, the choice of the renormalized parameter is not limited to $\bar{u}_{0}$. Any vertex function proportional to $\bar{u}_{0}$ in the perturbative region (for example a four-point vertex function calculated at some value of the set of its external momenta) is an acceptable choice provided that the differential momentum-scale dependence along $T_{1}$ be properly accounted for.

\section{E. Other infra-red attractive submanifolds in three dimensions}

\section{1. "Unusual" infra-red attractive submanifold in three dimensions}

The trajectories already drawn in fig. 3 show that $T_{1}$, which emerges from $P_{\mathrm{G}}$, is infra-red attractive for initial points chosen not very far from $P_{\mathrm{G}}$. But, by considering initial points chosen farther away from $P_{\mathrm{G}}$ in $\mathcal{S}_{\mathrm{c}}$, another infra-red attractive one-dimensional submanifold is evidenced [see fig. 6].

Let us call $T_{1}^{\prime}$ this new one-dimensional attractive submanifold. Which of the two submanifolds $T_{1}$ and $T_{1}^{\prime}$, corresponds to the slowest flow in the vicinity of $f^{*}$ ? Answering this question requires to considering the expected analytic expression of the approach to $f^{*}$ in $\mathcal{S}_{\mathrm{c}}$. It reads:

$$
\begin{aligned}
& r_{0}(l) \stackrel{l}{ } \stackrel{\rightarrow}{\simeq} r_{0}^{*}+b_{r}^{(1)} \exp \left(-l \omega_{1}\right) \\
&+b_{r}^{(2)} \exp \left(-2 l \omega_{1}\right)+\cdots, \\
& u_{0}(l) \stackrel{l}{ } \stackrel{\infty}{\simeq} u_{0}^{*}+b_{u}^{(1)} \exp \left(-l \omega_{1}\right) \\
& \\
&+b_{u}^{(2)} \exp \left(-2 l \omega_{1}\right)+\cdots, \\
& \ldots
\end{aligned}
$$

with $\omega_{1}>0$. The value of the exponent $\omega_{1}$ is characteristic of the degree of (infra-red) stability of the submanifold considered. We choose to denote by $\omega_{1}^{\prime}$ the exponent associated with $T_{1}^{\prime}$ and by $\omega_{1}$ that associated with $T_{1}$. The determination of $\omega_{1}$ (or $\left.\omega_{1}^{\prime}\right)$ from Eq. (3.11) is made by considering the effective exponent:

$$
\omega_{\mathrm{eff}}=-\frac{1}{r_{0}(l)-r_{0}^{*}} \frac{d r_{0}(l)}{d l}
$$

Note that a similar definition of $\omega_{\text {eff }}$ could have been written down in terms of $u_{0}(l)$ or $v_{0}(l)$ etc.

The limit of Eq. (3.23) as $l \rightarrow \infty$ gives $\omega_{1}$ or $\omega_{1}^{\prime}$ according to whether the flow approaches $f^{*}$ along $T_{1}$ or along $T_{1}^{\prime}$.

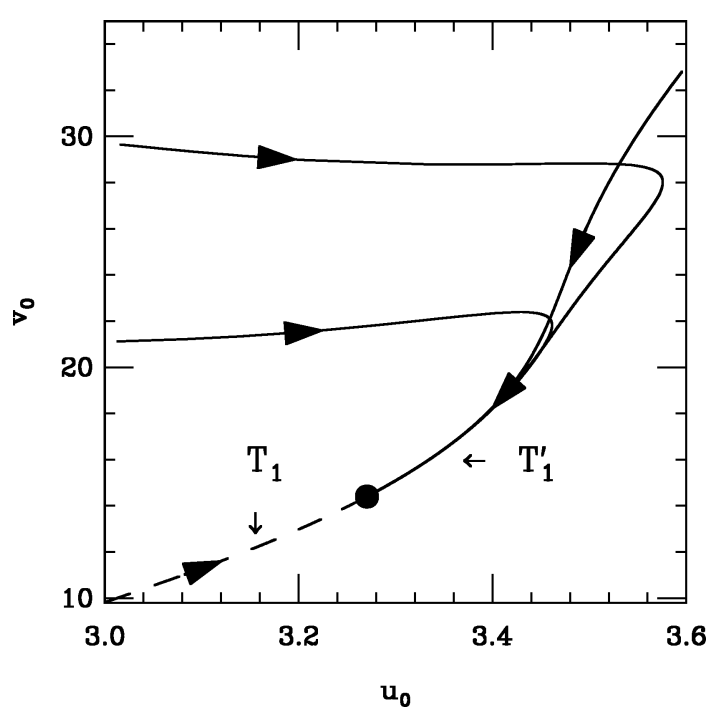

Fig. 6. The "unusual" infra-red stable one-dimensional submanifold in $\mathcal{S}_{\mathrm{c}}\left(T_{1}^{\prime}\right)$ obtained from Eq. (3.11) with $d=3$ (projection onto the plane $\left(u_{0}, v_{0}\right)$ ). The full circle represents the infra-red stable fixed point $f^{*}$ and the usual "renormalized trajectory" $T_{1}$ emerging from $P_{\mathrm{G}}$ is partially drawn (dashed curve). Arrows indicate the infra-red direction.

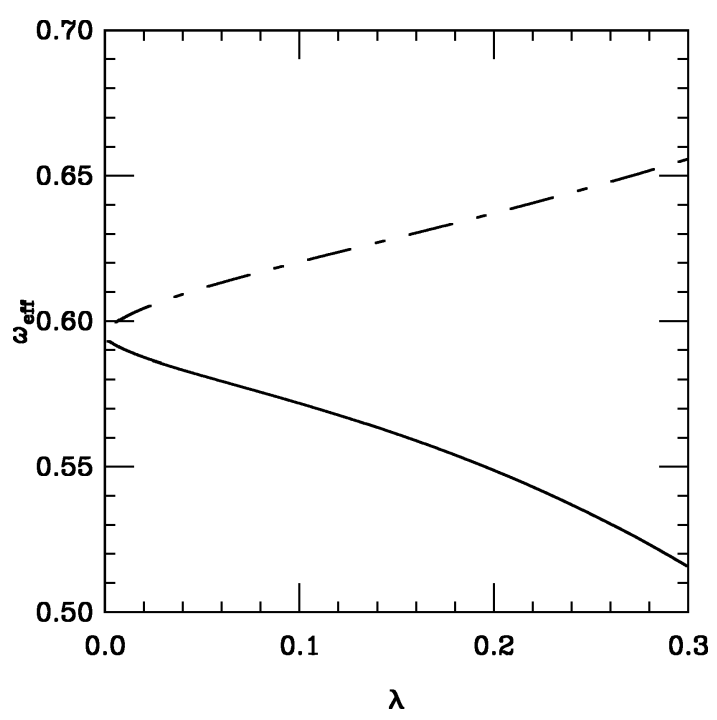

Fig. 7. Effective correction-exponent $\omega_{\text {eff }}(l)$ (Eq. (3.23) with $l=-\ln \lambda$ ) along $T_{1}$ (full curve) and along $T_{1}^{\prime}$ (dot-dashed curve). The estimate of the value of $\omega$ is $0.5955 \pm 0.0025$. 
Fig. 7 reproduces our determination of $\omega_{\text {eff }}$ both along $T_{1}$ and $T_{1}^{\prime}$. It shows that the two flows are equally slowly running. But the approach to $\omega \sim 0.6$ obtained on $T_{1}^{\prime}$ is "from above" while on $T_{1}$, it is "from below".

By comparison with the usual field-theoretic framework, we are led to associate the differential momentumscale dependence of the renormalized parameter $u$ with the one-dimensional submanifold of $\mathcal{S}_{\mathrm{c}}$ formed by $T_{1} \cup T_{1}^{\prime}$. The case $u<u_{\mathrm{ir}}^{*}$ corresponds to $T_{1}$ and the case $u>u_{\mathrm{ir}}^{*}$ to $T_{1}^{\prime}$.

Of course this strict reference to the "infinite cutoff limit" defined right on $T_{1}^{\prime}$ is formal on the field-theoretic point of view since no ultra-violet stable fixed point exists in association with $T_{1}^{\prime}$. Consequently, stricto-sensu, the "continuum limit" does not exist for $u>u_{\mathrm{ir}}^{*}$. Nevertheless, due to the 'large river' effect, the notion of effective field theory applies and gives a meaning [28] to (approximated) calculations performed in the analytical framework for $u>u_{\mathrm{ir}}^{*}[28,40]$. The acceptable accuracy of such calculations from the side $u>u_{\mathrm{ir}}^{*}$ is well illustrated by fig. 7 on which we see that the exponent $\omega$ (characteristic of $T_{1}$ ) may also be determined from the "wrong" side (along $T_{1}^{\prime}$ ).

The status of the infra-red attractive submanifold $T_{1}^{\prime}$ is interesting in that several statistical systems - especially the spin- $\frac{1}{2}$ Ising model in three dimensions correspond to this kind of approach to $f^{*}[27,6]$.

\section{Infra-red attractive submanifolds of lower degrees}

By studying how the approach to $f^{*}$ (along $T_{1}$ or $T_{1}^{\prime}$ ) depends on the initial point chosen in $\mathcal{S}_{\mathrm{c}}$, we find it possible to go continuously from one kind of approach $\left(T_{1}\right)$ to the other $\left(T_{1}^{\prime}\right)$. This means that, for any given value of $v_{0}(0)$ (with, say, $w_{0}(0)=\cdots=0$ ), there exists one value $u_{0 c}\left[v_{0}(0)\right]$ [67] for which the resulting trajectory in $\mathcal{S}_{\mathrm{c}}$ (i.e. obtained by adjusting also $r_{0}(0)$ to $\left.r_{0 \mathrm{c}}\left[u_{0 \mathrm{c}}\left\{v_{0}(0)\right\}, v_{0}(0)\right]\right)$ flows toward $f^{*}$ without having any point in common with either $T_{1}$ or $T_{1}^{\prime}$.

Let $\mathcal{S}_{\mathrm{c}}^{(2)} \subset \mathcal{S}_{\mathrm{c}}$ be the hyper-surface which, at $f^{*}$, is orthogonal to $T_{1} \cup T_{1}^{\prime}$. $\mathcal{S}_{\mathrm{c}}^{(2)}$ has one dimension less than $\mathcal{S}_{\mathrm{c}}$. Thus, by adjusting simultaneously two Hamiltonianparameters in $\mathcal{S}: r_{0 \mathrm{c}}(0)$ - to be in $\mathcal{S}_{\mathrm{c}}$ - and $u_{0 \mathrm{c}}(0)-$ to be in $\mathcal{S}_{\mathrm{c}}^{(2)}$ - we observe the presence of two new infrared attractive one-dimensional submanifolds (or slowly running flows) along which, however, the flows go faster than along $T_{1}$ and $T_{1}^{\prime}$ (otherwise they would have been observed first in $\mathcal{S}_{\mathrm{c}}$ ).

By studying the Wilson flows in $\mathcal{S}_{\mathrm{c}}^{(2)}$ we observe the attractive submanifolds $T_{2}$ and $T_{2}^{\prime}$ drawn in fig. 8. As in the preceding case, we have defined an effective exponent $\omega_{2 \mathrm{eff}}$ and observed that it tends to a unique value [68] $\left(\omega_{2} \sim 2.8\right)$ as $l \rightarrow \infty$ both along $T_{2}$ (from below) and $T_{2}^{\prime}$ (from above).

The existence of $T_{2}$ and $T_{2}^{\prime}$ suggests again the existence of another submanifold $\mathcal{S}_{\mathrm{c}}^{(3)} \subset \mathcal{S}_{\mathrm{c}}^{(2)}$ in which the approach to $f^{*}$ reveals two infra-red attractive submanifolds $T_{3}$ and $T_{3}^{\prime}$ with a same exponent $\omega_{3}>\omega_{2}$ and

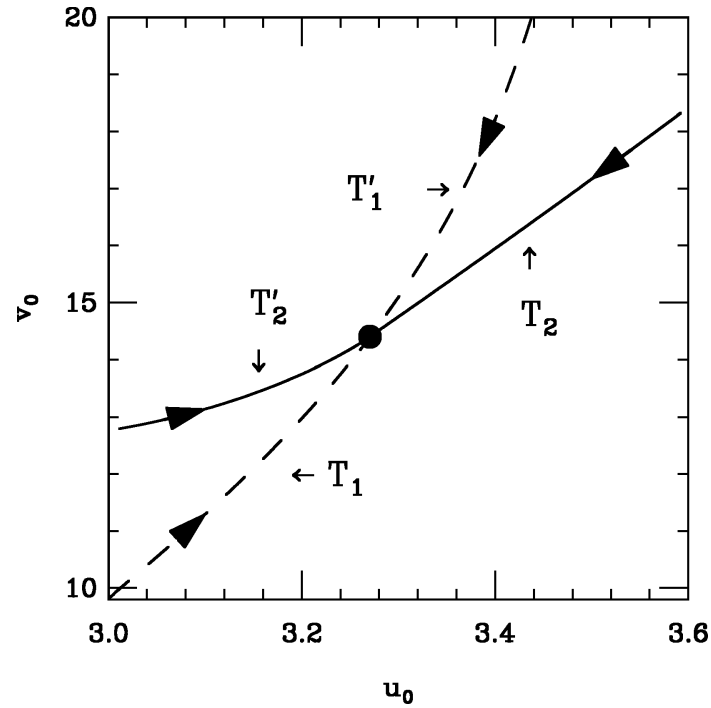

Fig. 8. The two infra-red attractive submanifolds in $\mathcal{S}_{\mathrm{c}}^{(2)}$ $T_{2}$ and $T_{2}^{\prime}$ (full curves) of same degree of stability but lower than on $T_{1}$ and on $T_{1}^{\prime}$ (dashed curves) (projection onto the plane $\left.\left(u_{0}, v_{0}\right)\right)$. The initial values chosen are $r_{0 \mathrm{c}}(0)=$ $-0.5832891 \cdots, u_{0}(0)=6.6615188 \cdots, v_{0}(0)=0\left(T_{2}\right)$ and $r_{0 c}(0)=-0.42788517 \cdots, u_{0}(0)=2, v_{0}(0)=26.13624\left(T_{2}^{\prime}\right)$.

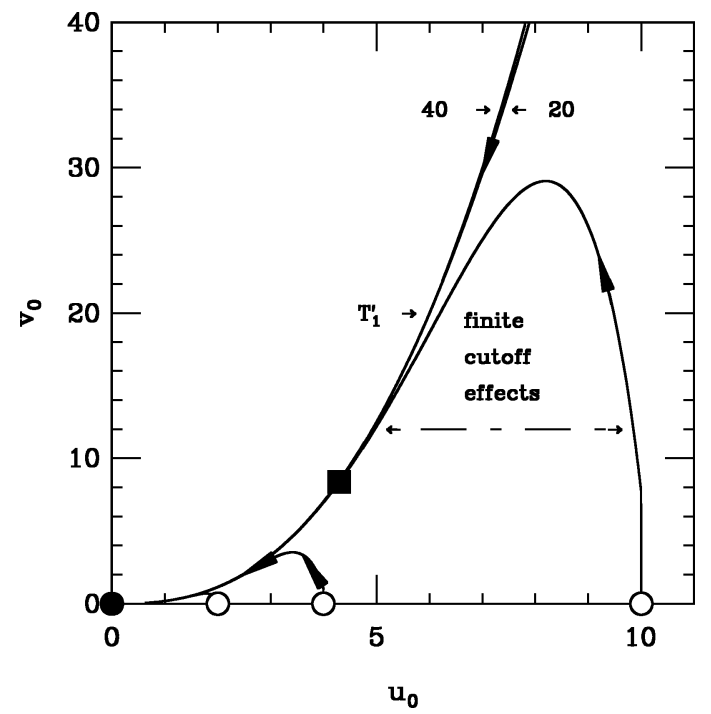

Fig. 9. Wilson's flows on the critical surface $\mathcal{S}_{\mathrm{c}}$ obtained from integration of Eq. (3.11) at $d=4$ (projection onto the plane $\left(u_{0}, v_{0}\right)$ ). Open circles indicate the initial points chosen on the canonical surface of $\mathcal{S}_{\mathrm{c}}:\left(u_{0}(0), r_{0 \mathrm{c}}(0)\right)=(2,-0.035806374 \cdots) ; \quad(4$, $-0.068682647 \cdots) ;(10,-0.1577796 \cdots)$. Two flows initialized at $(20,-0.2892822265 \cdots)$ and $(40,-0.52207031 \cdots)$ are partially reproduced. The full circle represents the infra-red stable fixed point $P_{\mathrm{G}}$. Arrows indicate the infra-red direction. The square roughly indicates where the effective field theory could make sense for the Wilson trajectory initialized at $u_{0}(0)=10$.

again one could define $\mathcal{S}_{\mathrm{c}}^{(4)} \subset \mathcal{S}_{\mathrm{c}}^{(3)}$ and find $\omega_{4}>\omega_{3}$ and so forth... 
At $f^{*}$, the directions orthogonal to $\mathcal{S}_{\mathrm{c}}, \mathcal{S}_{\mathrm{c}}^{(2)}, \mathcal{S}_{\mathrm{c}}^{(3)}$, $\mathcal{S}_{\mathrm{c}}^{(4)}, \cdots$ define a complete set of "scaling axes" which the attractive submanifolds $T_{n} \cup T_{n}^{\prime}(n=0,1,2, \cdots)$ are respectively tangent to. They correspond to the complete set of eigenfunctions, solutions of Eq. (3.11) linearized about $f^{*}$. It is well known that $f^{*}$ possesses one positive eigenvalue corresponding to the direction of infra-red instability (or relevant direction) orthogonal to $\mathcal{S}_{\mathrm{c}}$ (corresponding to the massive sector not considered here) and infinitely many negative eigenvalues associated with irrelevant directions (in $\mathcal{S}_{\mathrm{c}}$, orthogonal respectively to $\left.\mathcal{S}_{\mathrm{c}}^{(2)}, \mathcal{S}_{\mathrm{c}}^{(3)}, \mathcal{S}_{\mathrm{c}}^{(4)}, \cdots\right)$. These latter directions may be ordered according to the magnitude of the corresponding eigenvalues (related to the order $\omega<\omega_{2}<\omega_{3}<\cdots$ ). The scaling axes have, with respect to $f^{*}$, the same status as the axes of $\mathcal{S}$ with respect to the Gaussian fixed point $P_{\mathrm{G}}$.

As in the case of $T_{1}$ and $T_{1}^{\prime}$, we may associate, (at least up to some distance to $f^{*}$ ) a (formal) "renormalized" parameter to each of those infra-red stable submanifolds $T_{n}$ (or $T_{n}^{\prime}$ ). The universal differential momentum-scale dependences (independent of the initial point chosen in the basin of attraction of the "renormalized trajectory" of interest) associated to the infinite set of submanifolds $T_{n}$ - or $T_{n}^{\prime}$ - are distinguishable from each other by their asymptotic rate of flow which may be characterized by the universal asymptotic-exponents $\omega_{n}$. In the analytical treatment those new "renormalized" parameters are associated to the renormalization of the insertions of "composite operators".

\section{F. The four dimensional case}

The study of Eq. (3.11) at $d=4$ follows the same line as in $d=3$. The main difference is that the nontrivial fixed point $f^{*}$ coincides with $P_{\mathrm{G}}$ which becomes (marginally) infra-red stable in $\mathcal{S}_{\mathrm{c}}$. Consequently the massless renormalized trajectory $T_{1}$ (in $\mathcal{S}_{\mathrm{c}}$ ) shrinks to a point $\left(P_{\mathrm{G}}\right)$ but $T_{1}^{\prime}$ subsists (see fig. 9 ). Due to the lack of ultra-violet stable fixed point in $\mathcal{S}_{\mathrm{c}}$, the "continuum limit" of field theory does not exist but owing to the "large river effect" one may refer to the notion of effective field theory. The detailed discussion is similar to that of the three dimensional case for $T_{1}^{\prime}$ and will not be repeated in detail here. The reader may find supplementary considerations in $[17,69]$ for instance.

\section{THE SINGULARITIES OF THE PERTURBATION FRAMEWORK}

Three kinds of singularity are encountered in the analytical version of scalar field theory:

1. the "renormalons" that occur in the $\phi_{4}^{4}$-theory [70] and prevent the (renormalized) perturbation series from being Borel summable.

2. the infra-red singularities of the massless $\phi_{d}^{4}$-theory with $d<4[2,37]$.
3. the singularities at the infra-red stable fixed point $u_{\mathrm{ir}}^{*}($ for $d<4)[3,4]$.

The above-listed singularities are peculiar to the analytical treatment. They emerge from the realization of the following double request:

A the reduction of infinitely many parameters to very few renormalized (scale dependent) parameters,

B the possibility of pushing the momentum scale of reference up to infinity (existence of an ultra-violet stable fixed point).

These two requirements are explicit (and strictly not dissociated [71] ) in the Wilson process of defining the continuum limit of field theory (see part III). In the subtraction program of perturbation theory, however, both the infinite number of degrees of freedom and the existence of an ultra-violet stable fixed point are not truly considered explicitly [72].

In the renormalization program of perturbation theory, the renormalized parameter is implicitly defined as the parameter having in charge to exclusively follow the slowest flow in its complete extension (including, especially implicitly, the infinite momentum scale of reference). Indeed, the subtraction of the large-cutoff dependences amounts to selecting the slowest RG flow (only the definition of the renormalized parameter that exhibits the slowest flow may vary, see section III D 3).

The momentum scale dependence of the renormalized scalar coupling involves two aspects that explain the emergence of the above-listed singularities:

- the functional form of the momentum scale dependence (the account for infinitely many degrees of freedom)

- the domain of variation of the scale of reference ranging from $\infty$ to 0 .

We have already mentioned that the functional form of the momentum-scale dependence of $u$ has a nonperturbative nature: a reference to a supplementary dimensioned parameter $\Lambda_{\mathrm{r}}[73]$ is required to write: $u\left(\mu / \Lambda_{\mathrm{r}}\right)$. In order to get some idea on the nonperturbative form expected for $\Lambda_{\mathrm{r}}$ let us integrate Eq. (1.3) with the formal assumption that the initial condition is the existence of the "fundamental" invariant-scale $\Lambda_{\mathrm{r}}$. Hence, the offhand "definition" of $\Lambda_{\mathrm{r}}$ :

$$
\frac{\mu}{\Lambda_{\mathrm{r}}}=\exp \left[\int^{u} \frac{d x}{\beta(x)}\right]
$$

in which the undefinite integral stands for the primitive of the integrand, the additive constant being (by brute force) incorporated in the definition of $\Lambda_{\mathrm{r}}$. The knowledge of $\Lambda_{\mathrm{r}}$ then would univocally determines the value of $u$ which is actually associated to $\mu$.

For Eq. (4.24) to make some sense, an explicit analytic expression of $\beta(x)$ is required. Let us take $\beta(x)$ from the 
$1 / n$-expansion [74] with $\epsilon=4-d>0$, Eq. (4.24) then reads:

$$
\frac{\mu}{\Lambda_{\mathrm{r}}}=\left|\frac{\epsilon-u}{u}\right|^{1 / \epsilon}\left[1+O\left(\frac{1}{n}\right)\right]
$$

Performing the limit $\epsilon \rightarrow 0$ in Eq. (4.25), we obtain at $d=4$ :

$$
\frac{\mu}{\Lambda_{\mathrm{r}}}=e^{-1 / u}\left[1+O\left(\frac{1}{n}\right)\right]
$$

Those (obviously non-perturbative) expressions [Eqs. $(4.25,4.26)]$ are obtained by brute force but they suggest the possible emergence of pathological effects in perturbation theory. Those effects are the emergence of the above singularities 2 .

It is known that the perturbation expansion of vertex functions for the (super-renormalizable) massless scalarfield theory (with $d<4$ ) involves infra-red divergences at rational values of $d$. However, it has been shown [37] that theory "develops by itself" an infinite number of nonperturbative terms that are adapted to make the theory well defined at any $d$. Among those non-perturbative terms is the "critical-mass" parameter $m_{0 c}$. Beside the mathematics, these terms provide us with nothing but the coordinates (in a space of infinite dimension) of an (arbitrary) initial point lying on $T_{1}$. Indeed, $m_{0 \mathrm{c}}^{2}$ is similar to the initial critical value $r_{0 \mathrm{c}}(0)$ encountered in part III, the difference is that the former implicitly refers to some point of $T_{1}$ (entirely plunged in the Wilson space of infinite dimension hence the appearance of the other non-perturbative contributions) while the latter is associated with a chosen initial point of known coordinates (lying outside from $T_{1}$ ).

We may say that singularities 2 reflect the lack, in perturbation theory, of any specification of an initial point lying on $T_{1}$. It is not well known that those singularities are also present in four dimensions (for the massless theory). Indeed, Symanzik has shown [37] that $m_{0 \mathrm{c}}$, calculated within the framework of dimensional regularization $(\epsilon=4-d>0)$, takes on the following form:

$$
m_{0 \mathrm{c}}^{2}=g_{0}^{2 / \epsilon} h(\epsilon, n)
$$

in which $g_{0}$ is the dimensioned unrenormalized $\phi^{4}$ coupling and the function $h(\epsilon, n)$ displays poles at $\epsilon=$ $2 / k(k=2,3,4, \ldots)$. By considering how the minimal subtraction scheme of the (dimensionally regularized) $\phi_{4}^{4}$ theory works within the $1 / n$ expansion, Rim and Weisberger [75] discovered that the subtraction functions of perturbation theory (defined by the requirement of subtracting the simple poles located at $\epsilon=0$ and which introduces the renormalized parameter $u$ associated to the scale $\mu$ ) were not sufficient to obtain a well defined theory in the limit $\epsilon \rightarrow 0$. A new "mass-counter-term" (compared to the perturbative treatment), with a coefficient $\delta m^{2}$ defined as:

$$
\delta m^{2}=\mu^{2} \tilde{h}(\epsilon) e^{\frac{2}{u}}
$$

should be added to the dimensionally regularized "hamiltonian". This term arises as a consequence of poles at rational values of $\epsilon(\epsilon=2 / k, k=2,3, \ldots)$ which accumulate to give an essential singularity at $d=4$. This latter singularity, involved in $\tilde{h}(\epsilon)$, could not be eliminated by the standard subtraction of simple poles at $\epsilon=0$. Clearly, $\delta m^{2}$ bears some resemblance with $m_{0 \mathrm{c}}^{2}$. Indeed, Rim and Weisberger's result may be seen as Symanzik's result continued to $\epsilon \rightarrow 0$ at which dimension, the "infinite-cutoff limit" of perturbation theory cannot be effectuated without "renormalizing the coupling constant" [76].

In order to show the close relation of these "additionnal" terms with the "invariant scale" $\Lambda_{\mathrm{r}}$, we have applied the rules of dimensional renormalization on the critical parameter $r_{0 \mathrm{c}}$ calculated at order $1 / n$. Denoting by $m_{0 \mathrm{c}}^{2}$ the result, we obtain for $\epsilon>0(d<4)[26]$ :

$$
\begin{aligned}
& \frac{m_{0 \mathrm{c}}^{2}}{\mu^{2}}=-\frac{2}{n f(\epsilon)}\left(\frac{u f(\epsilon)}{u_{\mathrm{ir}}^{*}-u}\right)^{2 / \epsilon} \frac{\pi}{\sin \left(\frac{2 \pi}{\epsilon}\right)} \\
& +O\left(\frac{1}{n^{2}}\right) \quad \text { with } u<u_{\mathrm{ir}}^{*}
\end{aligned}
$$

in which

$$
f(\epsilon)=\Gamma(d / 2) \Gamma(1+\epsilon / 2)[\Gamma(1-\epsilon / 2)]^{2} / \Gamma(2-\epsilon)
$$

( $\Gamma(x)$ is the Euler gamma-function), and $u_{\mathrm{ir}}^{*}=\epsilon$ in the approximation considered.

Eq. (4.29) is a non-perturbative expression the analytic continuation to $\epsilon \leq 2$ of which displays poles at $\epsilon=2 / k(k=1,2,3, \ldots)$ which accumulate to give an essential singularity at $\epsilon=0$.

In order to allow the limit $\epsilon \rightarrow 0$ to be considered in the coefficient of the essential singularity of Eq. (4.29) it is necessary to first perform an analytic continuation of Eq. (4.29) to $u>u_{\mathrm{ir}}^{*}$. Using the relation $(-1)^{2 / \epsilon}=\cos \left(\frac{2 \pi}{\epsilon}\right)+i \sin \left(\frac{2 \pi}{\epsilon}\right)$, we obtain:

$$
\begin{aligned}
& \frac{m_{0 \mathrm{c}}^{2}}{\mu^{2}}=-\frac{2}{n f(\epsilon)}\left(\frac{u f(\epsilon)}{u-u_{\mathrm{ir}}^{*}}\right)^{2 / \epsilon} \pi\left[\cot \left(\frac{2 \pi}{\epsilon}\right)+i\right] \\
& +O\left(\frac{1}{n^{2}}\right) \quad \text { with } u>u_{\mathrm{ir}}^{*} .
\end{aligned}
$$

The limit $\epsilon \rightarrow 0$ performed in the coefficient of $\cot \left(\frac{2 \pi}{\epsilon}\right)$ of Eq. (4.30) leads to the "mass-counter-term" $\frac{1}{2} \delta m^{2} \phi^{2}$ found by Rim and Weisberger [75] with:

$$
\delta m^{2} \propto \mu^{2} e^{\frac{2}{u}} \pi \cot \left(\frac{2 \pi}{\epsilon}\right)
$$


For $\epsilon>0$, Eqs. $(4.29,4.30)$ provide the complete nonperturbative expression of Symanzik's critical-mass parameter in the "infinite-cutoff limit", at order $1 / n$ and corresponding to the submanifolds $T_{1}$ (Eq. (4.29)) and $T_{1}^{\prime}$ (Eq. (4.30)) encountered in part III.

By comparing the $(u, \mu)$-dependence of Eqs. $(4.29,4.30,4.31)$ to that of Eqs. $(4.25,4.26))$, we see that the non-perturbative properties of $\Lambda_{\mathrm{r}}$ are intimately related to the non-perturbative properties of the massless field theory. Moreover $m_{0 c}$ carries the same $u$-dependence as the "fundamental" invariant scale $\Lambda_{\mathrm{r}}$ itself. But the difference is that $m_{0 c}$ is absolutely needed (generated by the theory itself [37]) while $\Lambda_{\mathrm{r}}$ is a purely formal quantity attached to the artificial simple function $u(\lambda)$.

We have seen in part III that the functional form of the momentum scale dependence on $T_{1}$ is degenerated (section III C 2) and that it is the specification of the initial value $r_{0 c}(0)$ which allows us to specify a unique renormalization flow running "on" $T_{1}$. Similarly, the parameter $m_{0 c}$ allows us to specify which determination of the functional form of the momentum-scale dependence is actually referred to on $T_{1}$ (or on $T_{1}^{\prime}$ ).

With the massive theory, the singularities 2 disappear but the singularities 1 remain. Although the functional form of the scale dependence is again responsible for the emergence of those singularities, the mechanism is different from the previous case.

The renormalon singularities may be "removed" by considering all "composite operators" of dimension 8, 10 , etc. . [77,78] or, what is equivalent, by reintroducing an ultra-violet cutoff $[56,78]$. It has been shown, for instance, that the perturbative series in powers of $u_{0}(0)$ of the (unrenormalized) vertex functions of the $\phi_{4}^{4}$ - "infrared" model [79] are Borel summable [80].

Indeed, the renormalons appear because the trajectory $T_{1}^{\prime}$ does not take its source at an ultra-violet stable fixed point. Consequently, the associated domain of momentum scales covered by the running parameter $u$ is incomplete: when $u$ "reaches" infinity, the momentum scale of reference is still finite. Here is the problem: we do not know how to associate a genuine momentum scale of reference to a value of $u$ because there are infinitely many parameters to be initialized. When $u$ is the relevant parameter at a fixed point then this association is easy to do: each degree of freedom delegates one's powers to $u$. But, when there is no ultra-violet stable fixed point, one must actually specify the complete coordinates of an initial point lying on $T_{1}^{\prime}$, what is actually impossible. The other possibility is to consider some Wilson trajectory which will approach the trajectory $T_{1}^{\prime}$ in reducing the "cutoff", this is the $\phi_{4}^{4}$ - "infra-red" model mentioned above.

The inverse Borel transforms of the perturbative series of the $\phi_{4}^{4}$-vertex functions are made ambiguous due to branch-point singularities located at real positive values of the Borel variable $b$ (the "renormalon"-singularities). This does not imply that a Borel resummation of the series cannot be performed but rather that one does not know how to choose the integration contour in the complex $b$-plane so as to pick up such and such determination of the integrand at each singularity. Thus an infinite number of conditions, unknown in the analytic treatment, must be (re-)specified in order to fix one determination among an infinite number of allowed determinations (the coordinates of the initial point on $T_{1}^{\prime}$ associated to the arbitrarily chosen momentum scale of reference must be specified).

Notice that, if the above considerations are correct, the emergence of renormalon singularities is strictly linked to the specification of an initial point lying on $T_{1}^{\prime}$. If, on a pure mathematical ground (without any consideration to a well defined field theory), we exclusively limit our interest to the renormalization flow running on $T_{1}^{\prime}$ via the simple function $u(\lambda)$, then it would be possible to imagine a peculiar procedure to construct a $\beta$-function the perturbation series of which would be Borel summable.

In our view, this peculiar procedure corresponds to the so-called minimal subtraction scheme. In such a subtraction scheme, nothing else than the reference to a (specific) scale dependence defines the renormalized coupling constant $u$, in particular, no reference to a vertex function (involving the problem of controlling infinitely many degrees of freedom) is introduced contrary to what is done when using a subtraction point procedure.

The above remarks, that result from the discussions presented in the preceding parts, illustrate what, indeed, was already expected in four dimensions. Namely:

"that it is possible to define renormalization schemes such that the renormalization group functions $[\beta(u), \eta(u), \cdots]$ do not have ultra-violet renormalons" [78].

The arguments presented in this section allow us to propose candidates for those schemes: the minimal subtraction schemes.

As for the singularities 3, they emerge for the same reason as the renormalon singularities appear in four dimensions: the lack of ultra-violet stable fixed point associated with the trajectory $T_{1}^{\prime}$ (i.e., in the range $u>u_{\mathrm{ir}}^{*}$ ).

In view of testing some basical assumptions of the field theoretic approach to critical phenomena, a latticeanalog of the renormalized $\phi^{4}$ coupling constant has been defined as follows [81]:

$$
u_{\text {latt }}=-\xi^{-d}\left[\frac{\partial^{2} \chi}{\partial H^{2}}\right] \chi^{-2}
$$

in which $\xi$ and $\chi$ are respectively, the correlation length and the susceptibility of the $\phi^{4}$-lattice-model; $H$ is the magnetic field. So defined $u_{\text {latt }}$ "looks like" the renormalized $\phi^{4}$ coupling constant $u$ of the massive scheme in which the renormalized mass $m$ is essentially replaced by $\xi^{-1}$.

By analogy with field theory, the lattice analog of the $\beta$-function is defined as follows [3,4]:

$$
\beta_{\text {latt }}\left(u_{\text {latt }}\right)=\left[-\epsilon \frac{d}{d u_{\text {latt }}} \ln \left(\tilde{\xi}\left(u_{\text {latt }}\right)\right)^{\epsilon}\right]^{-1}
$$


in which $\tilde{\xi}=g_{0}^{\frac{1}{\epsilon}} \xi$ with $g_{0}$ the dimensioned unrenormalized $\phi^{4}$ coupling constant and $\epsilon=4-d$.

Nickel's discovery of confluent singularities in the $\beta$ function follows from the remark that renormalization theory predicts [61] several kinds of confluent critical singularities. The approach of $u_{\text {latt }}$ to $u_{\mathrm{ir}}^{*}$ as $\tilde{\xi} \rightarrow \infty$ is, thus, controlled by the following equation:

$$
\begin{aligned}
u_{\text {latt }}= & u_{\mathrm{ir}}^{*}\left[1+a_{1} \tilde{\xi}^{-\omega_{1}}+a_{2} \tilde{\xi}^{-2 \omega_{1}}+\cdots\right. \\
& +b_{1} \tilde{\xi}^{-\omega_{2}}+b_{2} \tilde{\xi}^{-2 \omega_{2}}+\cdots \\
& \left.+\cdots+c_{1} \tilde{\xi}^{-\frac{1}{\nu}}+c_{2} \tilde{\xi}^{-\frac{2}{\nu}}+\cdots\right]
\end{aligned}
$$

in which the exponents $\omega_{n}$ have already been encountered in sections III D and III E 2. Supplementary terms proportional to $c_{n}$ account for analytic confluent corrections in temperature usually not explicitly considered by renormalization theory and thus not relevant to the present discussion.

Now, on reverting Eq. (4.34), and neglecting the above-mentioned analytic confluent corrections in temperature, one obtains:

$$
\begin{aligned}
\tilde{\xi} \simeq & a_{0}\left(u_{\mathrm{ir}}^{*}-u_{\mathrm{latt}}\right)^{-1 / \omega_{1}}\left[1+a_{2}^{\prime}\left(u_{\mathrm{ir}}^{*}-u_{\mathrm{latt}}\right)+\cdots\right. \\
& \left.+b_{1}^{\prime}\left(u_{\mathrm{ir}}^{*}-u_{\mathrm{latt}}\right)^{\left(\omega_{2}-\omega_{1}\right) / \omega_{1}}+\cdots\right]
\end{aligned}
$$

and finally, using Eq. (4.33):

$$
\begin{aligned}
\beta_{\text {latt }}\left(u_{\text {latt }}\right) \simeq & \omega_{1}\left(u_{\text {latt }}-u_{\text {ir }}^{*}\right)+d_{1}\left(u_{\mathrm{ir}}^{*}-u_{\text {latt }}\right)^{2}+\cdots \\
& +d_{2}\left(u_{\text {ir }}^{*}-u_{\text {latt }}\right)^{\Delta_{2} / \Delta_{1}}+\cdots
\end{aligned}
$$

in which $\Delta_{n}=\omega_{n} \nu$.

Obviously, the second line of Eq. (4.36) displays a leading confluent-branch-point-singularity that is not accounted for in the resummation procedure of $\beta(u)$ as used in [82]. Nickel then makes reference to the work of Golner and Riedel [83] who found $\Delta_{2} \simeq 2 \Delta_{1}$ (see also the more recent work of Newman and Riedel [84] that yields in addition $\Delta_{3} \simeq 3 \Delta_{1}$ ) to argue that the confluent singularities could be practically very weak as explicitly assumed later on by Zinn-Justin [85]. If so, Nickel does not understand

"... why the confluent terms in the lattice-analog $\beta_{\text {latt }}\left(u_{\text {latt }}\right)$ can apparently not be ignored" in an actual numerical analysis of high-temperature series.

At the light of the preceding parts, that may well be understood.

The confluent singularities found by Nickel in $\beta_{\text {latt }}$ are due to what we have called the "finite-cutoff effects" (see section IIID 1). These effects "measure" the significant deviations, in the critical surface $\mathcal{S}_{\mathrm{c}}$, between a simple Wilson trajectory (of a lattice or "cutoff" theory) and the ideal "trajectory" $T_{1}$ (or $T_{1}^{\prime}$ ) of part III. Consequently, in the field theoretic framework, Nickel's confluent singularities within the $\beta$-function are either absent $\left(u<u_{\mathrm{ir}}^{*}\right.$, by definition of the "continuum limit") or are extremely weak $\left(u>u_{\mathrm{ir}}^{*}\right)$ due to the 'large river' effect [86].

The weakness of the confluent singularities could explain why, in their careful studies of the various available series, Le Guillou and Zinn-Justin [82] have not observed any numerical effect which could be clearly associated to the existence of such singularities.

Despite the supposed weakness of the confluent singularities in the range $u \geq u_{\mathrm{ir}}^{*}$ for the massive framework in three dimensions, attempts at systematically accounting for them have been made [4] (on the basis of the knowledge of seven orders instead of six orders only, except for the function $\beta(g)$ for which only the already known six orders were used). Their study has provided new exponent estimates which "are in better agreement with other model calculations". This result could be seen as a concrete confirmation of the presence of confluent singularities in the range $u \geq u_{\mathrm{ir}}^{*}$. But, due to the lack of relevant information on the actual value of $\Delta_{2}$ and on higher order terms in the series of $\beta(u)$, it has only been concluded that the previous error bars [82] could be "unrealistically small". It is also fair to indicate that, due to the lack of order in the series of $\beta(u)$ a bias could have been introduced in the determination of the fixed point value $u_{\mathrm{ir}}^{*}$.
[1] J. Zinn-Justin, Euclidean Field Theory and Critical Phenomena (Oxford University Press, 1989, second edition (1993), third edition (1996)).

[2] G. Parisi, in Cargèse Summer School (1973) unpublished and J. Stat. Phys. 23, 49 (1980).

[3] B. G. Nickel, in Phase Transitions, ed. by M. Lévy, J. C. Le Guillou and J. Zinn-Justin (Plenum, New-York and London, 1982).

[4] B. G. Nickel, Physica A177, 189 (1991).

[5] G. A. Baker, J. M. Kincaid, Phys. Rev. Lett. 42, 1431 (1979); J. Stat. Phys. 24, 469 (1981).

[6] A. Liu, M. E. Fisher, J. Stat. Phys. 58, 431 (1990).

[7] B. G. Nickel, Macromolecules 24, 1358 (1991).
[8] A. D. Sokal, Europhys. Lett. 27, 661 (1994).

[9] B. Li, N. Madras, A. D. Sokal, J. Stat. Phys. 80, 661 (1995).

[10] C. Bagnuls, C. Bervillier, Phys. Rev. Lett. 76, 4094 (1996); M. A. Anisimov, A. A. Povodyrev, V. D. Kulikov, J. V. Sengers, Phys. Rev. Lett. 75, 3146 (1995); ibid. 76, 4095 (1996).

[11] By analytical we mean a framework which controls a very limited number of parameters as in perturbation theory.

[12] M. Gell-Mann, F. E. Low, Phys. Rev. 95, 1300 (1954).

[13] E. Stueckelberg, A. Petermann, Helv. Phys. Acta 26, 499 (1953); L. V. Ovsyannikov, Dokl. Akad. Nauk SSSR 109, 1112 (1956); N. N. Bogoliubov, D. V. Shirkov, in 
Introduction to the Theory of Quantized Fields (Interscience, New-York, 1959); C. G. Callan, Phys. Rev. D 2, 1541 (1970); K. Symanzik, Comm. Math. Phys. 18, 227 (1970); A. Petermann, Phys. Rep. 53, 157 (1979).

[14] E. Brézin, J. C. Le Guillou, J. Zinn-Justin, in Phase Transitions and Critical Phenomena, Vol. VI, Ed. by C. Domb and M. S. Green (Academic Press, New-York, 1976).

[15] K. G. Wilson, J. Kogut, Phys. Rep. C 12, 77 (1974).

[16] J. F. Nicoll, T. S. Chang, H. E. Stanley, Phys. Rev. Lett. 33, 540 (1974).

[17] A. Hasenfratz, P. Hasenfratz, Nucl. Phys. B 270 [FS16], 687 (1986)

[18] G. Felder, Comm. Math. Phys. 111, 101 (1987).

[19] Usually called the " $\varphi^{4}$-theory".

[20] F. J. Wegner, A. Houghton, Phys. Rev. A 8, 401 (1973).

[21] J. Polchinski, Nucl. Phys. B 231, 269 (1984).

[22] J. Hughes, J. Liu, Nucl. Phys. B 307, 183 (1988).

[23] M. Lüscher, P. Weisz, Nucl. Phys. B 290 [FS20], 25 (1987).

[24] A. Margaritis, G. Ódor, A. Patkós, Z. Phys. C 39, 109 (1988); Yu. M. Ivanchenko, A. A. Lisyansky, A. E. Filippov, J. Phys. A 23, 91 (1990); Phys. Lett. A 150, 100 (1990); A. E. Filippov, S. A. Breus, Phys. Lett. A 158, 300 (1991); A. A. Lisyansky, Yu. M. Ivanchenko, A. E. Filippov, J. Stat. Phys. 66, 1667 (1992); A. E. Filippov, A. V. Radievskii, Sov. Phys-JETP 75, 1022 (1992); Phys. Lett. A 169, 195 (1992); JETP Lett. 56, 87 (1992); Yu. M. Ivanchenko, A. A. Lisyansky, Phys. Rev. A 45, 8525 (1992); S. A. Breus, A. E. Filippov, Physica A 192, 486 (1993); G. Zumbach, Phys. Rev. Lett. 71, 2421 (1993); Phys. Lett. A 190, 225 (1994); Nucl. Phys. B 413, 754 (1994); ibid. 771 (1994); P. E. Haagensen, Y. Kubyshin, J. I. Latorre, E. Moreno, Phys. Lett. B 323, 330 (1994); T. R. Morris, Int. J. Mod. Phys. A 8, 2411 (1994); Phys. Lett. B 329, 241 (1994); Phys. Lett. B 334, 355 (1994); Nucl. Phys. B 458 [FS], 477 (1996); A. E. Filippov, J. Stat. Phys. 75, 241 (1994); R. D. Ball, P. E. Haagensen, J. I. Latorre, E. Moreno, Phys. Lett. B 347, 80 (1995); S.-B. Liao, J. Polonyi, Phys. Rev. D 51, 4474 (1995); K. I. Aoki, K. Morikawa, W. Souma, J.-I. Sumi, H. Terao, Prog. Theor. Phys. 95, 409 (1996).

[25] C. Bagnuls, C. Bervillier, Saclay preprint T94/026 (1994), unpublished.

[26] C. Bagnuls, C. Bervillier, Phys. Rev. Lett. 60, 1464 (1988).

[27] C. Bagnuls, C. Bervillier, Phys. Rev. B 41, 402 (1990).

[28] C. Bagnuls, C. Bervillier, Phys. Lett. A 195, 163 (1994).

[29] Some authors may consider the renormalization as a simple technique to get rid of infinities in the perturbation field theory and some others as a technique to define the continuum limit of lattice field theories.

[30] Subtraction of infinities that occur at any order of the expansion in powers of $g_{0}$ of the unrenormalized (or regularized) $N$-point vertex functions $\Gamma^{(N)}\left(p_{i} ; m_{0}^{2}, g_{0}, \Lambda_{0}\right)$ in the limit $\Lambda_{0} \rightarrow \infty$ at fixed $m_{0}$ and $g_{0}$ when $d=4$.

[31] G. 't Hooft, M. Veltman, Nucl. Phys. B 44, 189 (1972); J. F. Ashmore, Lett. Nuovo Cimento 4, 289 (1972); C. G. Bollini, J. J. Giambiagi, Phys. Lett. B 40, 566 (1972); G. 't Hooft, Nucl. Phys. B 61, 455 (1973); P. Butera, G. M. Cicuta, E. Montaldi, in Renormalization and Invariance in QFT, p. 1, ed. by E. R. Caianello (Plenum Press, New-York and London, 1974).
[32] S. Weinberg, Phys. Rev. D 8, 3497 (1973).

[33] F. Dyson, Phys. Rev. 85, 631 (1952); C. S. Lam, Nuovo Cimento 55, 258 (1968); L. N. Lipatov, Zh. Eksp. Teor. Fiz. 72, 411 (1977) [Sov. Phys.-JETP 45, 216 (1977)]; Zh. Eksp. Teor. Fiz. 25, 116 (1977) [JETP Lett. 25, 104 (1977)].

[34] J. P. Eckmann, J. Magnen, R. Sénéor, Comm. Math. Phys. 39, 251 (1975).

[35] In four dimensions the perturbative series involves "renormalon" singularities that do not allow Borel summability (see part IV).

[36] K. G. Wilson, M. E. Fisher, Phys. Rev. Lett. 28, 240 (1972).

[37] K. Symanzik, Nuovo Cimento Lett. 8, 771 (1973); G. Parisi, Nucl. Phys. B 150, 163 (1979); R. Jackiw, S. Templeton, Phys. Rev. D 23, 2291 (1981); M. C. Bergère, F. David, Ann. Phys. (New-York) 142, 416 (1982).

[38] However Baker and Kincaid did not make any distinction between the Wilson and field theoretic versions of the renormalization theory (see [27]).

[39] D. J. Gross, in Methods in Field Theory, ed. by $R$. Balian and J. Zinn-Justin (North Holland, Amsterdam, 1976); D. J. Amit, Field Theory. The Renormalization Group and Critical Phenomena (World Scientific, Singapore, 1984); G. Parisi, Statistical Field Theory (AddisonWesley Publ., 1988); M. Le Bellac, Des Phénomènes Critiques aux Champs de Jauge (Inter Editions/Editions du CNRS, 1988); C. Itzykson, J. M. Drouffe, Statistical Field Theory, Vol. I (Cambridge University Press, Cambridge, 1989).

[40] B. Krüger, L. Schäfer, J. Phys. I (France) 4, 757 (1994); L. Schäfer, Phys. Rev. E 50, 3517 (1994).

[41] D. J. Gross, in Recent Developments in Quantum Field Theory, ed. by J. Ambjorn, B. J. Durhuus and J. L. Petersen (Elsevier Science Publishers B. V., 1985).

[42] For technical reasons: the removal of infinities occuring at any order of perturbation theory.

[43] $u(1)=u$ is associated to the momentum-scale $\mu$ while $u(\lambda)$ is associated to the momentum-scale $\lambda \mu$.

[44] D. V. Shirkov, Int. J. Mod. Phys. A 3, 1321 (1988).

[45] J. F. Nicoll, T. S. Chang, H. E. Stanley, Phys. Rev. B 12, 458 (1975).

[46] K. G. Wilson, Adv. Math. 16, 444 (1975).

[47] F. Dyson, Comm. Math. Phys. 12, 91 (1969).

[48] Only one hamiltonian-parameter has to be adjusted since $\mathcal{S}_{\mathrm{c}}$ has the dimension of $\mathcal{S}$ minus one.

[49] For not too large values of the initial hamiltonian parameters.

[50] This choice is arbitrary, we could as well have chosen the plane $\left\{r_{0}, u_{0}\right\}$, for example.

[51] In the analytical treatment the limit of interest is that of infinite cutoff which is called the "continuum limit" when the ultra-violet cutoff is provided by the inverse of a lattice spacing.

[52] $\Lambda_{0}$ is then no longer a constant.

[53] Measured in the previously assumed fixed unit $\Lambda_{0}$, the second value of $\mu_{0}$ would be smaller than the previous one since $e^{-l_{1,3}} \Lambda_{0}<e^{-l_{2,3}} \Lambda_{0}$.

[54] In the present study, we find $\omega_{1} \simeq 0.6$ (see section III E 1).

[55] Symanzik's program for improving lattice field theories (based on perturbative considerations) relies upon this 
idea that the limit of interest in field theory consists in choosing the initial hamiltonian right on $T_{1}$. Although the number of conditions to be imposed on the lattice (or unrenormalized) hamiltonian is infinite in principle, it appears to be finite at each order of perturbation.

[56] K. Symanzik, in New Developments in Quantum Field Theory and Statistical Mechanics, ed. by M. Lévy and P. K. Mitter (Plenum, New-York, 1977); in Recent Development in Gauge Field Theories, ed. by G 't Hooft et al. (Plenum, New-York, 1980); in Mathematical Problems in Theoretical Physics. Lecture Notes in Physics, ed. by R. Schrader et al. (Springer, Berlin, 1982); Nucl. Phys. B 226, 205 (1983); Nucl. Phys. B 226, 187 (1983).

[57] The Gaussian fixed point is the only exactly accessible point of $T_{1}$. But initializing the renormalization process exactly at $P_{\mathrm{G}}$ would not produce any information on the flow running along $T_{1}$ (by definition of a fixed point).

[58] Due to the infinity of parameters involved in the "exact" RG treatment, the limit of field theory does not actually correspond to the removal of $\Lambda_{0}$ which is the seed of the momentum unit.

[59] A. E. Filippov, JETP Lett. 60, 141 (1994).

[60] Our numerical study of Eq. (3.11) is not accurate enough to correctly account for the earlier transitory regimes, hence the vertical dashed lines in the early parts of Wilson's trajectories drawn on fig. 3 .

[61] F. J. Wegner, Phys. Rev. B 5, 4529 (1972); in Phase Transitions and Critical Phenomena Vol. VI, ed. by C. Domb and M. S. Green (Academic Press, New-York, 1976).

[62] Due to the local potential approximation, our estimate of $\omega_{1}$ (see section IIIE 1 ) differs from the usual value of $\omega$ quoted in [1].

[63] H. M. Georgi, in The New Physics, ed. by P. Davies (Cambridge University Press, 1989); G. P. Lepage, in From Actions to Answers, proc. of the 1989 TASI Summer School, ed. by T. De Grand and D. Toussaint (World Scientific, Singapore, 1990).

[64] The correct definition of the renormalized parameter is rather the relevant parameter at a once infra-red unstable fixed point [15]. But, in four dimensions the perturbatively renormalized $\phi^{4}$-coupling is (marginally) an irrelevant parameter for the gaussian fixed point (see section III F), hence the present definition.

[65] The relevance of a parameter is an important notion. A relevant parameter summarizes by itself the conspiracy of infinitely many degrees of freedom contributing to a field theory.

[66] The RG is a semi-group (the inverse transform is not defined) the action of which decreases the momentum-scale of reference.

[67] Our $u_{0 c}\left[v_{0}(0)\right]$ is identical to the $u_{0 c}\left[w_{0}(0)\right]$ introduced on p. 132 of [15].

[68] The determination of $\omega_{2}$ is made less accurate than that of $\omega_{1}$ because of the need for adjusting two hamiltonianparameters instead of only one.

[69] P. Hasenfratz, J. Nager, Z. Phys. C 37, 477 (1988).

[70] D. J. Gross, A. Neveu, Phys. Rev. D 10, 3235 (1974).

[71] They are linked together via the unique requirement that the renormalized parameter is the relevant parameter at a once infra-red unstable fixed point.

[72] The renormalizable character of a theory often appears as the consequence of a mathematical miracle.

[73] The "invariant scale" $\Lambda_{\mathrm{r}}$ is frequently introduced and used in studies of non-abelian gauge field theory for instance.

[74] A non-perturbative framework.

[75] C. Rim, W. I. Weisberger, D 32, 3244 (1985).

[76] Except the dimension $d$, the difference between Eq. (4.28) and Eq. (4.27) originates in the renormalization of the coupling constant.

[77] G. Parisi, Phys. Lett. B 76, 65 (1978); Phys. Rep. 49, 215 (1979).

[78] M. C. Bergère, F. David, Phys. Lett. B 135, 253 (1984).

[79] A massless " $\phi_{4}^{4}$ "-theory with a finite ultra-violet cutoff $\Lambda_{0}$ similar to that considered in part III.

[80] J. S. Feldman, J. Magnen, V. Rivasseau, R. Sénéor, Comm. Math. Phys. 109, 437 (1987); K. Gawedski, A. Kupiainen, Phys. Rev. Lett. 54, 92 (1985); Comm. Math. Phys. 99, 197 (1985).

[81] G. A. Baker, Jr, Phys. Rev. B 15, 1552 (1977).

[82] J. C. Le Guillou, J. Zinn-Justin, Phys. Rev. Lett. 39, 95 (1977); Phys. Rev. B 21, 3976 (1980); J. Phys. Lett. (France) 46, L137 (1985).

[83] G. R. Golner, E. K. Riedel, Phys. Lett. A 58, 1365 (1976).

[84] K. E. Newman, E. K. Riedel, Phys. Rev. B 30, 6615 (1984).

[85] J. Zinn-Justin, in Phase Transitions, ed. by M. Lévy, J. C. Le Guillou, J. Zinn-Justin (Plenum, New-York and London, 1982).

[86] The fact that one deals with the massive theory does not matter since we are more interested in the vicinity of $u_{\mathrm{ir}}^{*}$ where mass effects vanish.

\title{
ТЕОРЕТИКО-ПОЛЬОВІ МЕТОДИ В ДОСЛІДЖЕННІ КРИТИЧНИХ ЯВИЩ
}

\author{
К. Банюльс ${ }^{\dagger}$, К. Бервілье $\mathrm{e}^{\dagger \dagger}$ \\ ${ }^{\dagger}$ Відділ фізики конденсованого стану, ${ }^{\dagger \dagger}$ Відділ теоретичной фізики \\ C. Е. А.-Сакле, F91191 Жіф-сюр-Івет Седекс, Франиіл
}

Коротко проілюстровано, як теоретико-польовий підхід до критичних явищ працює в більш складній ренормалізаційній теорії Вільсона і якісно обговорюються межі його застосовності. Між іншим, ми пропонуємо, що диференціяльні ренормалізаційні функції (такі як $\beta$-функція) пертурбативної скалярної теорії у чотирьох вимірах $\left(\varphi_{4}^{4}\right)$ повинні бути такі, що підсумовуються за Борелем, за умови, що іх обчислено за схемою мінімального віднімання. 